\title{
FOURIER MULTIPLIERS, SYMBOLS, AND NUCLEARITY ON COMPACT MANIFIFOLDS
}

\author{
By \\ Julio Delgado ${ }^{1,3}$ And Michael RuZhansky ${ }^{2,3}$
}

\begin{abstract}
The notion of invariant operators, or Fourier multipliers, is discussed for densely defined operators on Hilbert spaces, with respect to a fixed partition of the space into a direct sum of finite-dimensional subspaces. As a consequence, given a compact manifold $M$ endowed with a positive measure, we introduce a notion of the operator's full symbol adapted to the Fourier analysis relative to a fixed elliptic operator $E$. We give a description of Fourier multipliers, or of operators invariant relative to $E$. We apply these concepts to study Schatten classes of operators on $L^{2}(M)$ and to obtain a formula for the trace of trace class operators. We also apply it to provide conditions for operators between $L^{p}$-spaces to be $r$-nuclear in the sense of Grothendieck.
\end{abstract}

\section{Introduction}

Let $M$ be a closed manifold (i.e., a compact smooth manifold without boundary) of dimension $n$ endowed with a positive measure $d x$. Given an elliptic positive pseudo-differential operator $E$ of order $v$ on $M$, by considering an orthonormal basis consisting of eigenfunctions of $E$, we associate a discrete Fourier analysis to the operator $E$ in the sense introduced by Seeley [See65], [See69]. This analysis allows us to introduce further a notion of invariant operators and of matrix-symbols corresponding to those operators. The operators on $M$ are then analysed in terms of the corresponding symbols relative to the operator $E$.

As a general framework, we first discuss invariant operators, or Fourier multipliers, in a general Hilbert space $\mathcal{H}$. This notion is based on a partition of $\mathcal{H}$ into a direct sum of finite-dimensional subspaces, so that a densely defined operator on $\mathcal{H}$ can be decomposed as acting in these subspaces. There are two main examples of this construction discussed in the paper: operators on $\mathcal{H}=L^{2}(M)$ for a compact

(C) The author(s) 2018. This article is published with open access at Springerlink.com

${ }^{1}$ Supported by Marie Curie IIF 301599 and by the Leverhulme Grant RPG-2014-02.

${ }^{2}$ Supported by EPSRC grant EP/K039407/1.

${ }^{3}$ No new data was collected or generated during the course of the research. 
manifold $M$ as well as operators on $\mathcal{H}=L^{2}(G)$ for a compact Lie group $G$. The difference in approaches to these settings is in the choice of partitions of $\mathcal{H}$ into direct sums of subspaces: in the former case, they are chosen as eigenspaces of a fixed elliptic pseudo-differential operator on $M$, while, in the latter case, they are chosen as linear spans of matrix coefficients of inequivalent irreducible unitary representations of $G$.

We note that for some results, the assumptions of self-adjointness and ellipticity of $E$ can be dropped; see [RT15].

We give applications of these notions to the derivation of conditions characterising those invariant operators on $L^{2}(M)$ that belong to Schatten classes. We also give conditions for nuclearity on $L^{p}$-spaces and, more generally, for the $r$ nuclearity of operators. While the theory of $r$-nuclear operators in general Banach spaces has been developed by Grothendieck [Gro55] with numerous further advances (e.g., in [HP10, Kön78, Olo72, Pie84, RL13]), in this paper we give conditions in terms of symbols for operators to be $r$-nuclear from $L^{p_{1}}(M)$ to $L^{p_{2}}(M)$ for $1 \leq p_{1}, p_{2}<\infty$ and $0<r \leq 1$. Consequently, we determine relations between $p_{1}, p_{2}, r$ and $\alpha$, ensuring that the powers $(I+E)^{-\alpha}$ are $r$-nuclear. Trace formulas are also obtained relating operator traces to expressions involving their symbols.

In the recent work [DR14c], the authors found sufficient conditions for operators to belong to Schatten classes $S_{p}$ on compact manifolds in terms of their Schwartz integral kernels. For $p<2$, it is customary to impose regularity conditions on the kernel because there are counterexamples to conditions formulated only in terms of the integrability of kernels. Such examples go back to Carleman's work [Car16], and their relevance to Schatten classes has been discussed in [DR14b]. A characteristic feature of conditions of this paper is that no regularity is assumed either on the symbol or on the kernel. In the case of compact Lie groups, our results extend results on Schatten classes and on $r$-nuclear operators on $L^{p}$ spaces that have been obtained in [DR13] and [DR14b]. We show this by relating the symbols introduced in this paper to matrix-valued symbols on compact Lie groups developed in [RT13] and in [RT10].

Schatten classes of pseudo-differential operators in the setting of the WeylHörmander calculus have been considered in [Tof06], [Tof08], [BN04], [BN07], [BT10]. Conditions for symbols of lower regularity are given in [Sob14]. For the global analysis of pseudo-differential operators on $\mathbb{R}^{n}$, see [BBR96]; see also [NR10, Chapter 4] for a basic general introduction to Schatten classes.

To formulate the notions more precisely, let $\mathcal{H}$ be a complex Hilbert space, and let $T: \mathcal{H} \rightarrow \mathcal{H}$ be a linear compact operator. Denote by $T^{*}: \mathcal{H} \rightarrow \mathcal{H}$ the adjoint of $T$. Then the linear operator $\left(T^{*} T\right)^{\frac{1}{2}}: \mathcal{H} \rightarrow \mathcal{H}$ is positive and compact. Let 
$\left(\psi_{k}\right)_{k}$ be an orthonormal basis for $\mathcal{H}$ consisting of eigenvectors of $|T|=\left(T^{*} T\right)^{1 / 2}$, and let $s_{k}(T)$ be the eigenvalue corresponding to the eigenvector $\psi_{k}, k=1,2, \ldots$ The non-negative numbers $s_{k}(T), k=1,2, \ldots$, are called the singular values of $T: \mathcal{H} \rightarrow \mathcal{H}$. If $0<p<\infty$ and the sequence of singular values is $p$-summable, then $T$ is said to belong to the Schatten class $S_{p}(\mathcal{H})$. It is well known that each $S_{p}(\mathcal{H})$ is an ideal in $\mathscr{L}(\mathcal{H})$. If $1 \leq p<\infty$, a norm is associated to $S_{p}(\mathcal{H})$ and is given by $\|T\|_{S_{p}}=\left(\sum_{k=1}^{\infty}\left(s_{k}(T)\right)^{p}\right)^{1 / p}$. If $1 \leq p<\infty$, the class $S_{p}(\mathcal{H})$, endowed with the norm $\|T\|_{S_{p}}$, becomes a Banach space. If $p=\infty$, we define $S_{\infty}(\mathcal{H})$ as the class of bounded linear operators on $\mathcal{H}$, with operator norm $\|T\|_{S_{\infty}}:=\|T\|_{o p}$. For the Schatten class $S_{2}$, we sometimes write $\|T\|_{\text {HS }}$ instead of $\|T\|_{S_{2}}$. In the case $0<p<1$, the quantity $\|T\|_{S_{p}}$ defines only a quasi-norm, and $S_{p}(\mathcal{H})$ is also complete. The space $S_{1}(\mathcal{H})$ is known as the trace class, and an element of $S_{2}(\mathcal{H})$ is usually called a Hilbert-Schmidt operator. For the basic theory of Schatten classes, we refer the reader to [GK69], [RS75], [Sim79], and [Sch70].

It is well known that the class $S_{2}\left(L^{2}\right)$ is characterised by the square integrability of the corresponding integral kernels. However, kernel estimates of this type are not effective for classes $S_{p}\left(L^{2}\right)$ with $p<2$. This is explained by a classical Carleman's example [Car16] on the summability of Fourier coefficients of continuous functions; see [DR14b] for a complete explanation of this fact. This obstruction explains the relevance of symbolic Schatten criteria, and here we clarify the advantage of the symbol approach with respect to this obstruction. With this approach, no regularity of the kernel need be assumed.

In Section 6, we discuss the relation of our approach to that of the global analysis on compact Lie groups. In particular, in the case of compact Lie groups, the Fourier coefficients can be arranged into a (square) matrix rather than in a column, and this leads to several simplifications. On general compact manifolds, this is not possible since the multiplicities $d_{j}$ need not all be squares of integers.

We introduce $\ell^{p}$-style norms on the space of symbols $\Sigma$, yielding discrete spaces $\ell^{p}(\Sigma)$ for $0<p \leq \infty$, normed for $p \geq 1$. Denoting by $\sigma_{T}$ the matrix symbol of an invariant operator $T$ provided by Theorem 4.1, we can characterise Schatten classes of invariant operators on $L^{2}(M)$ concisely by conditions

$$
T \in \mathscr{L}\left(L^{2}(M)\right) \Longleftrightarrow \sigma_{T} \in \ell^{\infty}(\Sigma),
$$

and for $0<p<\infty$,

$$
T \in S_{p}\left(L^{2}(M)\right) \Longleftrightarrow \sigma_{T} \in \ell^{p}(\Sigma) ;
$$

see (7.4) and (7.5). Here, the condition that $T$ is invariant means that $T$ is strongly commuting with $E$; see Theorem 4.1. On the level of the Fourier transform, this 
means that $\widehat{T f}(\ell)=\sigma(\ell) \widehat{f}(\ell)$ for a family of matrices $\sigma(\ell)$, i.e., $T$ assumes the familiar form of a Fourier multiplier.

In Theorem 2.1 below, we discuss the abstract notion of symbol for operators densely defined in a general Hilbert space $\mathcal{H}$ and give several alternative formulations for invariant operators, or for Fourier multipliers, relative to a fixed partition of $\mathcal{H}$ into a direct sum $\mathcal{H}=\bigoplus_{j} H_{j}$ of finite-dimensional subspaces. Consequently, in Theorem 2.3, we give the necessary and sufficient condition for bounded extendability of an invariant operator to $\mathscr{L}(\mathcal{H})$ in terms of its symbol, and, in Theorem 2.5, the necessary and sufficient condition for the operator to be in Schatten classes $S_{r}(\mathcal{H})$ for $0<r<\infty$, as well as the trace formula for operators in the trace class $S_{1}(\mathcal{H})$ in terms of their symbols.

As our subsequent analysis relies to a large extent on properties of elliptic pseudo-differential operators on $M$, in Sections 3 and 4, we specify this abstract analysis to the setting of operators densely defined on $L^{2}(M)$. The main difference is that we now adopt the Fourier analysis to a fixed elliptic positive pseudodifferential operator $E$ on $M$, contrary to the case of an operator $E_{o} \in \mathscr{L}(\mathcal{H})$ in Theorem 2.2.

The notion of invariance depends on the choice of the spaces $H_{j}$. Thus, in the analysis of operators on $M$, we take $H_{j}$ 's to be the eigenspaces of $E$. However, other choices are possible. For example, for $\mathcal{H}=L^{2}(G)$ for a compact Lie group $G$, choosing $H_{j}$ 's as linear spans of representation coefficients for inequivalent irreducible unitary representations of $G$, we make a link to the quantization of pseudo-differential operator on compact Lie groups as in [RT10]. These two partitions coincide when inequivalent representations of $G$ produce distinct eigenvalues of the Laplacian; for example, this is the case for $G=\operatorname{SO}(3)$. However, the partitions are different when inequivalent representations produce equal eigenvalues, which is the case, for example, for $G=\mathrm{SO}(4)$. For the more explicit example on $\mathcal{H}=L^{2}\left(\mathbb{T}^{n}\right)$ on the torus, see Remark 2.6. A similar choice could be made in other settings, producing a discrete spectrum and finite-dimensional eigenspaces, for example for operators in Shubin classes on $\mathbb{R}^{n}$; see Chodosh [Cho11] for the case $n=1$.

The concept analogous to Schatten classes in the setting of Banach spaces is the notion of $r$-nuclearity, introduced by Grothendieck [Gro55]. It has applications to questions of the distribution of eigenvalues of operators in Banach spaces. In the setting of compact Lie groups, these applications have been discussed in [DR14b], and they include conclusions on the distribution or summability of eigenvalues of operators acting on $L^{p}$-spaces. Another application is the Grothendieck-Lidskii formula, which is the formula for the trace of operators on $L^{p}(M)$. Once we have 
$r$-nuclearity, most of our further arguments are then purely functional analytic, so they apply equally well in the present setting of closed manifolds. Because of this, we omit the repetition of statements and refer the reader to [DR14b] for further such applications.

Some results of this paper have been announced in [DR14a]. Here we provide their proofs. We also include, given by Theorem 4.1(iv), a correction to the formulation of [DR14a, Theorem 3.1(iv)].

The paper is organised as follows. In Section 2, we discuss Fourier multipliers and their symbols in general Hilbert spaces. In Section 3, we associate a global Fourier analysis to an elliptic positive pseudo-differential operator $E$ on a closed manifold $M$. In Section 4, we introduce the class of operators invariant relative to $E$ as well as their matrix-valued symbols, and use this to characterise invariant operators in Schatten classes in Section 5. In Section 6, we relate the analysis developed so far to the analysis on compact Lie groups from [RT13], [RT10], and establish formula relating their matrix symbols in the case when $M$ is a compact Lie group. In particular, we show that left-invariant operators on compact Lie groups are invariant in our sense. In Section 7, we analyse the integral kernels of invariant operators on general closed manifolds. Finally, in Section 8, we apply our analysis to study $r$-nuclear operators on $L^{p}$-spaces.

Throughout the paper, $\mathbb{N}_{0}=\mathbb{N} \cup\{0\}$. Also $\delta_{j \ell}$ denotes the Kronecker delta, i.e.,

$$
\delta_{j \ell}= \begin{cases}1 & \text { for } j=\ell \\ 0 & \text { for } j \neq \ell\end{cases}
$$

\section{Fourier multipliers in Hilbert spaces}

In this section, we present an abstract set up to describe what we call invariant operators, or Fourier multipliers, acting on a general Hilbert space $\mathcal{H}$. We give several characterisations of such operators and their symbols. Subsequently, we apply these notions to describe several properties of the operators, in particular, their boundedness on $\mathcal{H}$, as well as the Schatten properties.

We note that direct integrals (sums in our case) of Hilbert spaces have been investigated in a much greater generality; see, e.g., Bruhat [Bru68], Dixmier [Dix96, Ch 2., §2], [Dix77, Appendix]. The setting required for our analysis is much simpler, so we prefer to adapt it specifically for consequent applications, also providing short proofs for our statements.

The main application of the constructions below is in the setting when $M$ is a compact manifold without boundary, $\mathcal{H}=L^{2}(M)$, and $\mathcal{H}^{\infty}=C^{\infty}(M)$, which 
is described in detail in Section 3. However, several facts can be more clearly interpreted in the setting of abstract Hilbert spaces, which is our set up in this section. With this particular example in mind, in the following theorem, we can think of $\left\{e_{j}^{k}\right\}$ as being an orthonormal basis given by eigenfunctions of an elliptic operator on $M$, and $d_{j}$ as being the corresponding multiplicities. However, we allow flexibility in grouping the eigenfunctions in order to be able to cover also the case of operators on compact Lie groups.

Theorem 2.1. Let $\mathcal{H}$ be a complex Hilbert space, and let $\mathcal{H}^{\infty} \subset \mathcal{H}$ be a dense linear subspace of $\mathcal{H}$. Let $\left\{d_{j}\right\}_{j \in \mathbb{N}_{0}} \subset \mathbb{N}$, and let $\left\{e_{j}^{k}\right\}_{j \in \mathbb{N}_{0}, 1 \leq k \leq d_{j}}$ be an orthonormal basis of $\mathcal{H}$ such that $e_{j}^{k} \in \mathcal{H}^{\infty}$ for all $j$ and $k$. Let $H_{j}:=\operatorname{span}\left\{e_{j}^{k}\right\}_{k=1}^{d_{j}}$, and let $P_{j}: \mathcal{H} \rightarrow H_{j}$ be the orthogonal projection. For $f \in \mathcal{H}$, we set $\widehat{f}(j, k):=\left(f, e_{j}^{k}\right)_{\mathcal{H}}$ and denote by $\widehat{f}(j) \in \mathbb{C}^{d_{j}}$ the column of $\widehat{f}(j, k), 1 \leq k \leq d_{j}$. Let $T: \mathcal{H}{ }^{\infty} \rightarrow \mathcal{H}$ be a linear operator. Then the following conditions are equivalent:

(A) $T\left(H_{j}\right) \subset H_{j}$ for each $j \in \mathbb{N}_{0}$;

(B) for each $\ell \in \mathbb{N}_{0}$ there exists a matrix $\sigma(\ell) \in \mathbb{C}^{d_{\ell} \times d_{\ell}}$ such that

$$
\widehat{T e_{j}^{k}}(\ell, m)=\sigma(\ell)_{m k} \delta_{j \ell} .
$$

for all $e_{j}^{k}$

(C) if, in addition, $e_{j}^{k}$ are in the domain of $T^{*}$ for all $j$ and $k$, then for each $\ell \in \mathbb{N}_{0}$, there exists a matrix $\sigma(\ell) \in \mathbb{C}^{d_{\ell} \times d_{\ell}}$ such that

$$
\widehat{T f}(\ell)=\sigma(\ell) \widehat{f}(\ell)
$$

for all $f \in \mathcal{H}^{\infty}$.

The matrices $\sigma(\ell)$ in $(\mathrm{B})$ and $(\mathrm{C})$ coincide. The equivalent properties $(\mathrm{A})-(\mathrm{C})$ follow from the condition

(D) For each $j \in \mathbb{N}_{0}$, we have $T P_{j}=P_{j} T$ on $\mathcal{H}^{\infty}$.

If, in addition, $T$ extends to a bounded operator $T \in \mathscr{L}(\mathcal{H})$, then $(\mathrm{D})$ is equivalent to (A)-(C).

Under the assumptions of Theorem 2.1, we have the direct sum decomposition

$$
\mathcal{H}=\bigoplus_{j=0}^{\infty} H_{j}, \quad H_{j}=\operatorname{span}\left\{e_{j}^{k}\right\}_{k=1}^{d_{j}}
$$

and $d_{j}=\operatorname{dim} H_{j}$. The two applications that we consider are with $\mathcal{H}=L^{2}(M)$ for a compact manifold $M$ with $H_{j}$ being the eigenspaces of an elliptic pseudodifferential operator $E$, or with $\mathcal{H}=L^{2}(G)$ for a compact Lie group $G$ with $H_{j}=$ $\operatorname{span}\left\{\xi_{k m}\right\}_{1 \leq k, m \leq d_{\xi}}$ for a unitary irreducible representation $\xi \in\left[\xi_{j}\right] \in \widehat{G}$. The 
difference is that in the first case, the eigenvalues of $E$ corresponding to $H_{j}$ 's are all distinct, while in the second case, the eigenvalues of the Laplacian on $G$ for which $H_{j}$ 's are the eigenspaces, may coincide. In Remark 2.6, we give an example of this difference for operators on the torus $\mathbb{T}^{n}$.

In view of properties $(\mathrm{A})$ and $(\mathrm{C})$, respectively, an operator $T$ satisfying any of the equivalent properties (A)-(C) in Theorem 2.1 is called an invariant operator, or a Fourier multiplier relative to the decomposition $\left\{H_{j}\right\}_{j \in \mathbb{N}_{0}}$ in (2.1). If the collection $\left\{H_{j}\right\}_{j \in \mathbb{N}_{0}}$ is fixed once and for all, we can just say that $T$ is invariant or a Fourier multiplier.

The family of matrices $\sigma$ is called the matrix symbol of $T$ relative to the partition $\left\{H_{j}\right\}$ and to the basis $\left\{e_{j}^{k}\right\}$. It is an element of the space $\Sigma$ defined by

$$
\Sigma=\left\{\sigma: \mathbb{N}_{0} \ni \ell \mapsto \sigma(\ell) \in \mathbb{C}^{d_{\ell} \times d_{\ell}}\right\} .
$$

A criterion for the extendability of $T$ to $\mathscr{L}(\mathcal{H})$ in terms of its symbol is given in Theorem 2.3.

For $f \in \mathcal{H}$, in the notation of Theorem 2.1, by definition we have

$$
f=\sum_{j=0}^{\infty} \sum_{k=1}^{d_{j}} \widehat{f}(j, k) e_{j}^{k}
$$

with convergence of the series in $\mathcal{H}$. Since $\left\{e_{j}^{k}\right\}_{j \geq 0}^{1 \leq k \leq d_{j}}$ is a complete orthonormal system on $\mathcal{H}$, for all $f \in \mathcal{H}$, we have the Plancherel formula

$$
\|f\|_{\mathcal{H}}^{2}=\sum_{j=0}^{\infty} \sum_{k=1}^{d_{j}}\left|\left(f, e_{j}^{k}\right)\right|^{2}=\sum_{j=0}^{\infty} \sum_{k=1}^{d_{j}}|\widehat{f}(j, k)|^{2}=\|\widehat{f}\|_{\ell^{2}\left(\mathbb{N}_{0}, \Sigma\right)}^{2},
$$

where we interpret $\widehat{f} \in \Sigma$ as an element of the space

$$
\ell^{2}\left(\mathbb{N}_{0}, \Sigma\right)=\left\{h: \mathbb{N}_{0} \rightarrow \prod_{d} \mathbb{C}^{d}: h(j) \in \mathbb{C}^{d_{j}} \text { and } \sum_{j=0}^{\infty} \sum_{k=1}^{d_{j}}|h(j, k)|^{2}<\infty\right\},
$$

and where we have written $h(j, k)=h(j)_{k}$. In other words, $\ell^{2}\left(\mathbb{N}_{0}, \Sigma\right)$ is the space of all $h \in \Sigma$ such that $\sum_{j=0}^{\infty} \sum_{k=1}^{d_{j}}|h(j, k)|^{2}<\infty$. We endow $\ell^{2}\left(\mathbb{N}_{0}, \Sigma\right)$ with the norm

$$
\|h\|_{\ell^{2}\left(\mathbb{N}_{0}, \Sigma\right)}:=\left(\sum_{j=0}^{\infty} \sum_{k=1}^{d_{j}}|h(j, k)|^{2}\right)^{1 / 2} .
$$

We note that the matrix symbol $\sigma(\ell)$ depends not only on the partition (2.1) but also on the choice of the orthonormal basis. Whenever necessary, we indicate the 
dependance of $\sigma$ on the orthonormal basis by writing $\left(\sigma,\left\{e_{j}^{k}\right\}_{j \geq 0}^{1 \leq k \leq d_{j}}\right)$; we also refer to $\left(\sigma,\left\{e_{j}^{k}\right\}_{j \geq 0}^{1 \leq k \leq d_{j}}\right)$ as the symbol of $T$. Throughout this section, the orthonormal basis is fixed, and, unless there is some risk of confusion, the symbols are denoted simply by $\sigma$. In the invariant language, as is clear from the proof of Theorem 2.1 , the transpose $\sigma(j)^{\top}=\left.T\right|_{H_{j}}$ of the symbol $\sigma$ is just the restriction of $T$ to $H_{j}$, which is well-defined in view of the property (A).

We also sometimes write $T_{\sigma}$ to indicate that $T_{\sigma}$ is an operator corresponding to the symbol $\sigma$. It is cl,ear from the definition that invariant operators are uniquely determined by their symbols. Indeed, if $T=0$, then $\sigma=0$ for every choice of orthonormal basis. Moreover, by taking $j=\ell$ in Theorem 2.1, B], we obtain the following formula for the symbol:

$$
\sigma(j)_{m k}=\widehat{T e_{j}^{k}}(j, m)
$$

for all $1 \leq k, m \leq d_{j}$. The formula (2.7) furnishes an explicit formula for the symbol in terms of the operator and the orthonormal basis. The definition of Fourier coefficients tells us that for an invariant operators $T$,

$$
\sigma(j)_{m k}=\left(T e_{j}^{k}, e_{j}^{m}\right)_{L^{2}(M)} .
$$

In particular, $\sigma_{I}(j)=I_{d_{j}}$, where $I$ is the identity operator and $I_{d_{j}} \in \mathbb{C}^{d_{j} \times d_{j}}$ is the identity matrix.

Before proving Theorem 2.1, let us establish a formula relating symbols with respect to different orthonormal basis. For orthonormal bases $\left\{e_{\alpha}\right\}$ and $\left\{f_{\alpha}\right\}$ of $\mathcal{H}$, we consider the unitary operator $U$ determined by $U\left(e_{\alpha}\right)=f_{\alpha}$. Then

$$
\left(T e_{\alpha}, e_{\beta}\right)_{\mathcal{H}}=\left(U T e_{\alpha}, U e_{\beta}\right)_{\mathcal{H}}=\left(U T U^{*} U e_{\alpha}, U e_{\beta}\right)_{\mathcal{H}}=\left(U T U^{*} f_{\alpha}, f_{\beta}\right)_{\mathcal{H}}
$$

Denoting by $\left(\sigma_{T},\left\{e_{\alpha}\right\}\right)$ the symbol of $T$ with respect to the orthonormal basis $\left\{e_{\alpha}\right\}$ and by $\left(\sigma_{U T U^{*}},\left\{f_{\alpha}\right\}\right)$ the symbol of $U T U^{*}$ with respect to the orthonormal basis $\left\{f_{\alpha}\right\}$, we have

$$
\left(\sigma_{T},\left\{e_{\alpha}\right\}\right)=\left(\sigma_{U T U^{*}},\left\{f_{\alpha}\right\}\right) .
$$

Thus, the equivalence relation of basis $\left\{e_{\alpha}\right\} \sim\left\{f_{\alpha}\right\}$ given by a unitary operator $U$ induces the equivalence relation on the set $\Sigma$ of symbols given by (2.9). In view of this, we can also think of the symbol as an element of the space $\Sigma / \sim$ with the equivalence relation given by (2.9).

We make another remark concerning Theorem 2.1 (C). We use the condition that $e_{j}^{k}$ are in the domain $\operatorname{Dom}\left(T^{*}\right)$ of $T^{*}$ in showing the implication $(\mathrm{B}) \Rightarrow(\mathrm{C})$. Since $e_{j}^{k}$, s give a basis in $\mathcal{H}$, and are all the $e_{j}^{k}$ are contained in $\operatorname{Dom}\left(T^{*}\right), \operatorname{Dom}\left(T^{*}\right)$ 
is dense in $\mathcal{H}$. In particular, by [RS80, Theorem VIII.1], $T$ must be closable (in part (C)). These conditions are not restrictive for the further analysis since they are satisfied in the natural applications of this paper.

The principal application of the notions above are as follows, except for in the sequel we need more general operators $E$ unbounded on $\mathcal{H}$. In order to distinguish from this general case, in the following theorem, we use the notation $E_{o}$.

Theorem 2.2. With the notation of Theorem 2.1 let $E_{o} \in \mathscr{L}(\mathcal{H})$ be a linear continuous operator with $H_{j}$ as its eigenspaces: $E_{o} e_{j}^{k}=\lambda_{j} e_{j}^{k}$ for each $j \in \mathbb{N}_{0}$ and all $1 \leq k \leq d_{j}$. Then equivalent conditions (A)-(C) imply

(E) $T E_{o} e_{j}^{k}=E_{o} T e_{j}^{k}$ for each $j \in \mathbb{N}_{0}$ and $1 \leq k \leq j$,

and if $\lambda_{j} \neq \lambda_{\ell}$ for $j \neq \ell$, then (E) is equivalent to Theorem 2.1 (A)-(C). Moreover, if $T$ extends to a bounded operator $T \in \mathscr{L}(\mathcal{H})$ then the equivalent properties (A)-(D) imply

(F) $T E_{o}=E_{o} T$ on $\mathcal{H}$,

and if also $\lambda_{j} \neq \lambda_{\ell}$ for $j \neq \ell$, then $(\mathbb{E})$ is equivalent to $(\mathrm{A})-(\mathrm{E})$.

For an operator $T=F\left(E_{o}\right)$, when it is well-defined by the spectral calculus,

$$
\sigma_{F\left(E_{o}\right)}(j)=F\left(\lambda_{j}\right) I_{d_{j}} .
$$

In fact, this is also then well-defined for a function $F$ defined on $\lambda_{j}$, with finite values which are, e.g., $j$-uniformly bounded (also for non self-adjoint $E_{o}$ ). We first prove Theorem 2.1.

Proof of Theorem 2.1. $\quad(\mathrm{A}) \Rightarrow$ (B). If $T$ satisfies condition $(\mathrm{A})$, we consider the matrix of $\left.T\right|_{H_{j}}: H_{j} \rightarrow H_{j}$ with respect to the orthonormal basis $\left\{e_{j}^{i}: 1 \leq i \leq d_{j}\right\}$ of $H_{j}$ and denote it by $\beta(j)$. Then $T e_{j}^{k}=\sum_{i=1}^{d_{j}} \beta(j)_{k i} e_{j}^{i}$. Consequently,

$$
\widehat{T e_{j}^{k}}(\ell, m)=\left(T e_{j}^{k}, e_{\ell}^{m}\right)=\beta(j)_{k m} \delta_{j \ell}=\beta(\ell)_{k m} \delta_{j \ell} .
$$

We take then $\sigma(\ell):=\beta(\ell)^{\top}$; it belongs to $\mathbb{C}^{d_{\ell} \times d_{\ell}}$ and satisfies $(\mathbb{B})$.

(B) $\Rightarrow$ (A). Since $e_{j}^{k} \in \mathcal{H}^{\infty}$, writing the series (2.3) for $T e_{j}^{k} \in \mathcal{H}$, we have

$$
T e_{j}^{k}=\sum_{\ell} \sum_{m=1}^{d_{\ell}} \widehat{T e_{j}^{k}}(\ell, m) e_{\ell}^{m}=\sum_{\ell} \sum_{m=1}^{d_{\ell}} \sigma(\ell)_{m k} \delta_{j \ell} e_{\ell}^{m}=\sum_{m=1}^{d_{\ell}} \sigma(j)_{m k} e_{j}^{m} \in H_{j}
$$

Since $\left\{e_{j}^{m}: 1 \leq m \leq d_{j}\right\}$ spans $H_{j}$, we obtain (A).

$(\mathrm{B}) \Rightarrow(\mathrm{C})$. We assume in addition that $e_{j}^{k}$ are in the domain of $T^{*}$ for all $j$ and $k$. We also assume that for each $\ell \in \mathbb{N}_{0}$, there exists a matrix $\sigma(\ell) \in \mathbb{C}^{d_{\ell} \times d_{\ell}}$ such that

$$
\widehat{T e_{j}^{k}}(\ell, m)=\sigma(\ell)_{m k} \delta_{j \ell}
$$


Now, if $f \in \mathcal{H}^{\infty}$, then $T f \in \mathcal{H}$; and, by the inversion formula (2.3), we have

$$
f=\sum_{j=0}^{\infty} \sum_{k=1}^{d_{j}} \widehat{f}(j, k) e_{j}^{k}
$$

Now, using this and the fact that all $e_{\ell}^{m}$ are in the domain of $T^{*}$, we have

$$
\begin{aligned}
\widehat{T f}(\ell, m) & =\left(T f, e_{\ell}^{m}\right)=\left(f, T^{*} e_{\ell}^{m}\right)=\left(\sum_{j=0}^{\infty} \sum_{k=1}^{d_{j}} \widehat{f}(j, k) e_{j}^{k}, T^{*} e_{\ell}^{m}\right) \\
& =\sum_{j=0}^{\infty} \sum_{k=1}^{d_{j}} \widehat{f}(j, k)\left(T e_{j}^{k}, e_{\ell}^{m}\right)=\sum_{j=0}^{\infty} \sum_{k=1}^{d_{j}} \widehat{f}(j, k) \widehat{T e_{j}^{k}}(\ell, m) \\
& =\sum_{j=0}^{\infty} \sum_{k=1}^{d_{j}} \widehat{f}(j, k) \sigma(\ell)_{m k} \delta_{j \ell}=\sum_{k=1}^{d_{j}} \sigma(\ell)_{m k} \widehat{f}(\ell, k),
\end{aligned}
$$

where we have also used (2.12). Hence $\widehat{T f}(\ell)=\sigma(\ell) \widehat{f}(\ell)$, yielding (C).

$(\mathrm{C}) \Rightarrow(\mathrm{B})$. If $\widehat{T f}(\ell)=\sigma(\ell) \widehat{f}(\ell)$, then

$$
\widehat{T e_{j}^{k}}(\ell, m)=\left(\sigma(\ell) \widehat{e}_{j}^{k}(\ell)\right)_{m}=\sum_{i=1}^{d_{j}} \sigma(\ell)_{m i} \widehat{e}_{j}^{k}(\ell, i)=\sum_{i=1}^{d_{j}} \sigma(\ell)_{m i} \delta_{j \ell} \delta_{k i}=\sigma(\ell)_{m k} \delta_{j \ell},
$$

which gives $(\mathrm{B})$, even without any assumptions on $T^{*}$.

(D) $\Rightarrow$ (A). We take $f \in H_{j}$. Then $P_{j} f \in H_{j}$ since $P_{j} f=f$, so that by assumption (D), we have $T f=T P_{j} f=P_{j} T f \in H_{j}$, implying (A).

(A) $\Rightarrow(D)$. For this part, we assume, in addition, that $T$ extends to a bounded operator $T \in \mathscr{L}(\mathcal{H})$. First we show that this together with $\mathbb{A}$ implies that $T\left(H_{j}^{\perp}\right)$ is orthogonal to $H_{j}$. For $g \in H_{j}^{\perp}$, we can write $g=\sum_{\ell \neq j} \sum_{k=1}^{d_{\ell}}\left(g, e_{\ell}^{k}\right) e_{\ell}^{k}$ with the convergence in $\mathcal{H}$, so that $T g=\sum_{\ell \neq j} \sum_{k=1}^{d_{\ell}}\left(g, e_{\ell}^{k}\right) T e_{\ell}^{k}$ with the convergence in $\mathcal{H}$, due to the boundedness of $T$ on $\mathcal{H}$. Since, by $(\mathrm{A}], T e_{\ell}^{k} \in H_{\ell} \subset H_{j}^{\perp}$ for $\ell \neq j$, we conclude that $T g$ is orthogonal to $H_{j}$.

Let now $f \in \mathcal{H}^{\infty}$. Writing $f=f_{1}+f_{2}$ with $f_{1}:=P_{j} f$ so that $f_{1} \in H_{j}$ and $f_{2} \in H_{j}^{\perp}$ are both in $\mathcal{H}^{\infty}$, we have $P_{j} T f=P_{j} T f_{1}+P_{j} T f_{2}=T f_{1}=T P_{j} f$, since the proved claim $P_{j} f_{2}=0$ implies that $P_{j} T f_{2}=0$.

We now continue with the proof of Theorem 2.2 when the basis $e_{j}^{k}$ corresponds to the eigenvectors of an operator $E_{o} \in \mathcal{L}(\mathcal{H})$.

Proof of Theorem 2.2. $\quad(\mathrm{A}) \Rightarrow(\mathrm{E})$. Let us fix some $e_{j}^{k}$. By $(\mathrm{A})$, we can write $T e_{j}^{k}=\sum_{i=1}^{d_{j}} \alpha_{i} e_{j}^{i}$ for some constants $\alpha_{i}$. Then

$$
E_{o} T e_{j}^{k}=E_{o} \sum_{i=1}^{d_{j}} \alpha_{i} e_{j}^{i}=\sum_{i=1}^{d_{j}} \alpha_{i} \lambda_{j} e_{j}^{i}=\lambda_{j} \sum_{i=1}^{d_{j}} \alpha_{i} e_{j}^{i}=\lambda_{j} T e_{j}^{k}=T \lambda_{j} e_{j}^{k}=T E_{o} e_{j}^{k},
$$


which shows (E).

(E) $\Rightarrow$ (A). It suffices to prove that $T e_{j}^{k} \in H_{j}$, since $\left\{e_{j}^{k}: 1 \leq k \leq d_{j}\right\}$ forms a basis of the finite-dimensional space $H_{j}$. We can assume that $T e_{j}^{k} \neq 0$, since otherwise there is nothing to prove. We recall that $E_{o} e_{j}^{k}=\lambda_{j} e_{j}^{k}$. Using $(\mathbb{E})$, we have $\lambda_{j} T e_{j}^{k}=T E_{o} e_{j}^{k}=E_{o} T e_{j}^{k}$. Hence $T e_{j}^{k} \in \mathcal{H}$ is a non-zero eigenvector of $E_{o}$ corresponding to the eigenvalue $\lambda_{j}$. Consequently, since $H_{j}$ are maximal eigenspaces corresponding to $\lambda_{j}$, we must have $T e_{j}^{k} \in H_{j}$.

$(E) \Rightarrow(E)$. Since we have already shown that $(\mathrm{A})-(\mathrm{C})$ imply $(\mathbb{E})$, it suffices to prove that $(\mathrm{E})$ implies $(\mathrm{F})$ under the additional assumption that $T \in \mathscr{L}(\mathcal{H})$. Let us write $S:=E_{o} \circ T, D:=T \circ E_{o}$ and let $f \in \mathcal{H}$. Under the assumptions, both $S$ and $D$ are bounded on $\mathcal{H}$; hence (2.3) implies

$$
S f=\lim _{N} \sum_{j=0}^{N} \sum_{k=1}^{d_{j}}\left(f, e_{j}^{k}\right) S e_{j}^{k}=\lim _{N} \sum_{j=0}^{N} \sum_{k=1}^{d_{j}}\left(f, e_{j}^{k}\right) D e_{j}^{k}=D f,
$$

with the convergent series in $\mathcal{H}$.

$(\mathrm{F}) \Rightarrow(\mathrm{A})$. We note that we require $T \in \mathscr{L}(\mathcal{H})$ in order for $T E_{o}$ and $E_{o} T$ to make sense on $\mathcal{H}$. It is clear that $(\mathbb{E})$ implies $(\mathbb{E})$; and, under the additional assumption that $\lambda_{j} \neq \lambda_{\ell}$ for $j \neq \ell$, we already know that $(\mathrm{A})-(\mathrm{C})$ and $(\mathrm{E})$ are equivalent. If $T$ is bounded on $\mathcal{H}$, then they are also equivalent to $(\mathbb{D})$.

We have the following criterion for the extendability of a densely defined invariant operator $T: \mathcal{H}^{\infty} \rightarrow \mathcal{H}$ to $\mathscr{L}(\mathcal{H})$, which was an additional hypothesis for properties $(\mathrm{D})$ and $(\mathrm{F})$. In the statements below, we fix a partition into $H_{j}$ 's as in (2.1), and the invariance refers to it.

Theorem 2.3. An invariant linear operator $T: \mathcal{H}^{\infty} \rightarrow \mathcal{H}$ extends to a bounded operator from $\mathcal{H}$ to $\mathcal{H}$ if and only if its symbol $\sigma$ satisfies

$$
\sup _{\ell \in \mathbb{N}_{0}}\|\sigma(\ell)\|_{\mathscr{L}\left(H_{\ell}\right)}<\infty
$$

Moreover,

$$
\|T\|_{\mathscr{L}(\mathcal{H})}=\sup _{\ell \in \mathbb{N}_{0}}\|\sigma(\ell)\|_{\mathscr{L}\left(H_{\ell}\right)}
$$

where $T$ also denotes the extended operator. 
Proof. We often abbreviate $\|\sigma(\ell)\|_{\mathscr{L}\left(H_{\ell}\right)}$ to $\|\sigma(\ell)\|_{o p}$. Let us first suppose that $\|\sigma(\ell)\|_{o p} \leq C$ for all $\ell \in \mathbb{N}_{0}$. By the Plancherel formula (2.4), we have

$$
\begin{aligned}
\|T f\|_{\mathcal{H}}^{2} & =\|\widehat{T f}\|_{\ell^{2}\left(\mathbb{N}_{0}, \Sigma\right)}^{2}=\sum_{\ell}\|\widehat{T f}(\ell)\|_{\ell^{2}\left(\mathbb{C}^{d_{\ell}}\right)}^{2}=\sum_{\ell}\|\sigma(\ell) \widehat{f}(\ell)\|_{\ell^{2}\left(\mathbb{C}^{d_{\ell}}\right)}^{2} \\
& \leq \sum_{\ell}\|\sigma(\ell)\|_{o p}^{2}\|\widehat{f}(\ell)\|_{\ell^{2}\left(\mathbb{C}^{\left.d_{\ell}\right)}\right.}^{2} \leq \sup _{\ell}\|\sigma(\ell)\|_{o p}^{2} \sum_{\ell}\|\widehat{f}(\ell)\|_{\ell^{2}\left(\mathbb{C}^{d_{\ell}}\right)}^{2} \\
& =\left(\sup _{\ell}\|\sigma(\ell)\|_{o p}\right)^{2}\|f\|_{\mathcal{H}}^{2} .
\end{aligned}
$$

Conversely, let us suppose that $T$ is bounded on $\mathcal{H}$. Then there exists a constant $C>0$ such that $\|T f\|_{\mathcal{H}} \leq C$ for all $f$ such that $\|f\|_{\mathcal{H}}=1$. We can take $C:=\|T\|_{\mathscr{L}(\mathcal{H})}$. Hence $\left.T\right|_{H_{j}}: H_{j} \rightarrow H_{j}$ is bounded, and $\left\|\left.T\right|_{H_{j}}\right\|_{\mathscr{L}\left(H_{j}\right)} \leq C$. On the other hand, let $\beta(j)$ denote the matrix of $\left.T\right|_{H_{j}}: H_{j} \rightarrow H_{j}$ with respect to the orthonormal basis $\left\{e_{j}^{i}: 1 \leq i \leq d_{j}\right\}$ of $H_{j}$, as in the proof of (A) implies (B) in Theorem 2.1. We consider an unitary operator $U: H_{j} \rightarrow \mathbb{C}^{d_{j}}$, which defines coordinates in $\mathbb{C}^{d_{j}}$ of vectors in $H_{j}$ with respect to the orthonormal basis $\left\{e_{j}^{k}: 1 \leq k \leq d_{j}\right\}$ of $H_{j}$. We also consider the operator $A(j): \mathbb{C}^{d_{j}} \rightarrow \mathbb{C}^{d_{j}}$ induced by the matrix $\beta(j)$. Then $\left.T\right|_{H_{j}}=U^{*} A(j) U$ and

$$
\|\sigma(j)\|_{o p}=\|\beta(j)\|_{o p}=\|A(j)\|_{o p}=\left\|\left.T\right|_{H_{j}}\right\|_{\mathscr{L}\left(H_{j}\right)} \leq C,
$$

completing the proof.

We also record the formula for the symbol of the composition of two invariant operators.

Proposition 2.4. If $S, T: \mathcal{H}^{\infty} \rightarrow \mathcal{H}$ are invariant operators with respect to the same orthonormal partition and such that the domain of $S \circ T$ contains $\mathcal{H}^{\infty}$, then $S \circ T: \mathcal{H}^{\infty} \rightarrow \mathcal{H}$ is also invariant with respect to the same partition. Moreover, if $\sigma_{S}$ denotes the symbol of $S$ and $\sigma_{T}$ denotes the symbols of $T$ with respect to the same orthonormal basis, then $\sigma_{S \circ T}=\sigma_{S} \sigma_{T}$, i.e., $\sigma_{S \circ T}(j)=\sigma_{S}(j) \sigma_{T}(j)$ for all $j \in \mathbb{N}_{0}$.

Proof. Recalling the definition of the composition of densely defined operators, we see that the domain of $S \circ T$ is the space of functions $f$ in the domain of $T$ such that $T f$ is in the domain of $S$, in which case we set $(S \circ T) f=S(T f)$. The hyothesis says that we are in the position to use Theorem 2.1. Applying Theorem 2.1 C) repeatedly, we have

$$
\widehat{(S \circ T)} f(j)=\widehat{S(T f)}(j)=\sigma_{S}(j) \widehat{T f}(j)=\sigma_{S}(j) \sigma_{T}(j) \widehat{f}(j),
$$

so $S \circ T$ is invariant by Theorem 2.1 C). 
We now apply the above notions to give a characterisation of Schatten classes of invariant operators in terms of their symbols.

Theorem 2.5. Let $0<r<\infty$. An invariant operator $T \in \mathscr{L}(\mathcal{H})$ with symbol $\sigma$ is in the Schatten class $S_{r}(\mathcal{H})$ if and only if $\sum_{\ell=0}^{\infty}\|\sigma(\ell)\|_{S_{r}\left(H_{\ell}\right)}^{r}<\infty$. Moreover,

$$
\|T\|_{S_{r}(\mathcal{H})}=\left(\sum_{\ell=0}^{\infty}\|\sigma(\ell)\|_{S_{r}\left(H_{\ell}\right)}^{r}\right)^{1 / r} .
$$

In particular, if $T$ is in the trace class $S_{1}(\mathcal{H})$, then

$$
\operatorname{Tr}(T)=\sum_{\ell=0}^{\infty} \operatorname{Tr}(\sigma(\ell))
$$

Proof. First, we claim that Schatten classes of invariant operators can be characterised in terms of the projections to the eigenspaces $H_{\ell}$ :

$$
\|T\|_{S_{r}(\mathcal{H})}^{r}=\sum_{\ell=0}^{\infty}\left\|\left.T\right|_{H_{\ell}}\right\|_{S_{r}\left(H_{\ell}\right)}^{r} .
$$

Let us prove (2.15). Since $\|T\|_{S_{r}}=\||T|\|_{S_{r}}$, we can assume without loss of generality that $T$ is positive definite. We first observe that $\lambda$ is an eigenvalue (singular value) of $T$ if and only if $\lambda$ is an eigenvalue (singular value) of $\left.T\right|_{H_{\ell(\lambda)}}$ for some $\ell(\lambda)$. Indeed, if $\lambda$ is an eigenvalue of $T$, there exists $\varphi_{\lambda} \in \mathcal{H} \backslash\{0\}$ such that $T \varphi_{\lambda}=\lambda \varphi_{\lambda}$. Using Theorem 2.1(D), we obtain $T P_{\ell} \varphi_{\lambda}=\lambda P_{\ell} \varphi_{\lambda}$ for every $\ell$. Since $\varphi_{\lambda} \neq 0$, there exists $\ell(\lambda)$ such that $P_{\ell(\lambda)} \varphi_{\lambda} \neq 0$. Consequently, $\lambda$ is the eigenvalue of $\left.T\right|_{H_{\ell(\lambda)}}=T P_{\ell(\lambda)}$.

Conversely, since $T\left(H_{\ell(\lambda)}\right) \subset H_{\ell(\lambda)}$, an eigenvalue of $\left.T\right|_{H_{\ell(\lambda)}}$ is also an eigenvalue of $T$. Therefore, we obtain (2.15).

Now, given (2.15), to prove (2.13), it suffices to check that

$$
\|\sigma(\ell)\|_{S_{r}\left(H_{\ell}\right)}=\left\|\left.T\right|_{H_{\ell}}\right\|_{S_{r}\left(H_{\ell}\right)} .
$$

To prove (2.16), we consider an unitary operator $U: H_{\ell} \rightarrow \mathbb{C}^{d_{\ell}}$, which defines coordinates in $\mathbb{C}^{d_{\ell}}$ of functions in $H_{\ell}$ with respect to the orthonormal basis $\left\{e_{\ell}^{k}: 1 \leq k \leq d_{\ell}\right\}$ of $H_{\ell}$. We also consider the operator $A(\ell): \mathbb{C}^{d_{\ell}} \rightarrow \mathbb{C}^{d_{\ell}}$ induced by the matrix $\left(\sigma_{T}(\ell)\right)^{\top}$. Then $\left.T\right|_{H_{\ell}}=U^{*} A(\ell) U$, and basic properties of Schatten quasinorms imply that

$$
\left\|\left.T\right|_{H_{\ell}}\right\|_{S_{r}\left(H_{\ell}\right)}=\|A(\ell)\|_{S_{r}\left(\mathbb{C}^{\left.d_{\ell}\right)}\right.}=\|\sigma(\ell)\|_{S_{r}},
$$

completing the proof of (2.16) and of (2.13). 
Finally, let us prove (2.14) for operators in the trace class $S_{1}(\mathcal{H})$. Since the trace $\operatorname{Tr}(T)$ does not depend on the choice of the orthonormal basis in $\mathcal{H}$, using property (C) and (2.11), we can write

$$
\begin{aligned}
\operatorname{Tr}(T) & =\sum_{\ell} \sum_{k=1}^{d_{\ell}}\left(T e_{\ell}^{k}, e_{\ell}^{k}\right)=\sum_{\ell} \sum_{k=1}^{d_{\ell}} \sum_{m=1}^{d_{\ell}} \sigma(\ell)_{m k}\left(e_{\ell}^{m}, e_{\ell}^{k}\right)=\sum_{\ell} \sum_{k=1}^{d_{\ell}} \sum_{m=1}^{d_{\ell}} \sigma(\ell)_{m k} \delta_{m k} \\
& =\sum_{\ell} \sum_{k=1}^{d_{\ell}} \sigma(\ell)_{k k}=\sum_{\ell} \operatorname{Tr}(\sigma(\ell)),
\end{aligned}
$$

completing the proof.

Remark 2.6. Membership in $\mathscr{L}(\mathcal{H})$ and in the Schatten classes $S_{r}(\mathcal{H})$ does not depend on the decomposition of $\mathcal{H}$ into subspaces $H_{j}$ as in (2.1). However, the notion of invariance does depend on it. For example, let $\mathcal{H}=L^{2}\left(\mathbb{T}^{n}\right)$ for the $n$-torus $\mathbb{T}^{n}=\mathbb{R}^{n} / \mathbb{Z}^{n}$. Choosing $H_{j}=\operatorname{span}\left\{e^{2 \pi \mathrm{i} j \cdot x}\right\}, j \in \mathbb{Z}^{n}$, we recover the construction of Section 6 on compact Lie groups. Moreover, invariant operators with respect to $\left\{H_{j}\right\}_{j \in \mathbb{Z}^{n}}$ are the translation invariant operators on the torus $\mathbb{T}^{n}$. However, to recover the construction of Section 4 on manifolds, we take $\widetilde{H}_{\ell}$ to be the eigenspaces of the Laplacian $E$ on $\mathbb{T}^{n}$, so that

$$
\widetilde{H_{\ell}}=\bigoplus_{|j|^{2}=\ell} H_{j}=\operatorname{span}\left\{e^{2 \pi \mathrm{i} j \cdot x}: j \in \mathbb{Z}^{n} \text { and }|j|^{2}=\ell\right\}, \quad \ell \in \mathbb{N}_{0} .
$$

Then translation invariant operators on $\mathbb{T}^{n}$, i.e., operators invariant relative to the partition $\left\{H_{j}\right\}_{j \in \mathbb{Z}^{n}}$, are also invariant relative to the partition $\left\{\widetilde{H}_{\ell}\right\}_{\ell \in \mathbb{N}_{0}}$ (or relative to the Laplacian, in terminology of Section 4). If we have information on the eigenvalues of $E$, like we do on the torus, we may sometimes also recover invariant operators relative to the partition $\left\{\widetilde{H}_{\ell}\right\}_{\ell \in \mathbb{N}_{0}}$ as linear combinations of translation invariant operators composed with phase shifts and complex conjugation.

\section{Fourier analysis associated to an elliptic operator}

Our main application is the study of operators on compact manifolds, so we start this section by describing the discrete Fourier series associated to an elliptic positive pseudo-differential operator as an adaptation of the construction in Section 2 . In order to fix the notation for the rest of the paper, we give some explicit expressions for notions of Section 2 in the present setting.

Let $M$ be a compact smooth manifold of dimension $n$ without boundary, endowed with a fixed volume element $d x$. We denote by $\Psi^{v}(M)$ the Hörmander class of pseudo-differential operators of order $v \in \mathbb{R}$, i.e., operators which, in every 
coordinate chart, are operators in Hörmander classes on $\mathbb{R}^{n}$ with symbols in $S_{1,0}^{v}$; see, e.g., [Shu01] or [RT10]. In this paper, we use the class $\Psi_{c l}^{v}(M)$ of classical operators, i.e., operators with symbols having (in all local coordinates) an asymptotic expansion of the symbol in positively homogeneous components; see, e.g., [Dui11]. Furthermore, we denote by $\Psi_{+}^{v}(M)$ the class of positive definite operators in $\Psi_{c l}^{v}(M)$, and by $\Psi_{e}^{v}(M)$ the class of elliptic operators in $\Psi_{c l}^{v}(M)$. Finally, $\Psi_{+e}^{v}(M):=\Psi_{+}^{v}(M) \cap \Psi_{e}^{v}(M)$ denotes the class of classical positive elliptic pseudodifferential operators of order $v$. We note that complex powers of such operators are well-defined; see, e.g., Seeley [See67]. In fact, all pseudo-differential operators considered in this paper are classical, so we may omit explicitly mentioning this every time. But we note that we could equally well work with general operators in $\Psi^{v}(M)$, since their powers have similar properties; see, e.g., [Str72].

Inspired by constructions considered by Seeley ([See65], [See69], see also Greenfield and Wallach [GW73]), we now associate a discrete Fourier analysis to the operator $E \in \Psi_{+e}^{v}(M)$ However, we adapt the construction to our purposes and, in the sequel, also prove several auxiliary statements concerning the eigenvalues of $E$ and their multiplicities that are useful to us in the subsequent analysis. In general, the construction below is exactly the one appearing in Theorem 2.1.

The eigenvalues of $E$ (counted without multiplicities) form a sequence $\left\{\lambda_{j}\right\}$, which we order so that

$$
0=\lambda_{0}<\lambda_{1}<\lambda_{2}<\cdots .
$$

To each eigenvalue $\lambda_{j}$ corresponds the finite-dimensional eigenspace $H_{j}$ of functions on $M$, which are smooth due to the ellipticity of $E$. We set $d_{j}:=\operatorname{dim} H_{j}$, and $H_{0}:=\operatorname{ker} E, \lambda_{0}:=0$. We also set $d_{0}:=\operatorname{dim} H_{0}$. Since the operator $E$ is elliptic, it is Fredholm, hence also $d_{0}<\infty$; see [Ati68], [Hör85a] for various properties of $H_{0}$ and $d_{0}$.

We fix an orthonormal basis of $L^{2}(M)$ consisting of eigenfunctions of $E$ :

$$
\left\{e_{j}^{k}\right\}_{j \geq 0}^{1 \leq k \leq d_{j}},
$$

where $\left\{e_{j}^{k}\right\}^{1 \leq k \leq d_{j}}$ is an orthonormal basis of $H_{j}$. Let $P_{j}: L^{2}(M) \rightarrow H_{j}$ be the corresponding projection. We denote by $(\cdot, \cdot)$ the inner product of $L^{2}(M)$. Observe that

$$
P_{j} f=\sum_{k=1}^{d_{j}}\left(f, e_{j}^{k}\right) e_{j}^{k}
$$

for $f \in L^{2}(M)$. The "Fourier" series takes the form

$$
f=\sum_{j=0}^{\infty} \sum_{k=1}^{d_{j}}\left(f, e_{j}^{k}\right) e_{j}^{k},
$$


for each $f \in L^{2}(M)$. The Fourier coefficients of $f \in L^{2}(M)$ with respect to the orthonormal basis $\left\{e_{j}^{k}\right\}$ is denoted by

$$
(\mathcal{F} f)(j, k):=\widehat{f}(j, k):=\left(f, e_{j}^{k}\right) .
$$

We call the collection of $\widehat{f}(j, k)$ the Fourier coefficients of $f$ relative to $E$, or simply the Fourier coefficients of $f$.

Since $\left\{e_{j}^{k}\right\}_{j \geq 0}^{1 \leq k \leq d_{j}}$ forms a complete orthonormal system in $L^{2}(M)$, we have the Plancherel formula (2.4), namely,

$$
\|f\|_{L^{2}(M)}^{2}=\sum_{j=0}^{\infty} \sum_{k=1}^{d_{j}}\left|\left(f, e_{j}^{k}\right)\right|^{2}=\sum_{j=0}^{\infty} \sum_{k=1}^{d_{j}}|\widehat{f}(j, k)|^{2}=\|\widehat{f}\|_{\ell^{2}\left(\mathbb{N}_{0}, \Sigma\right)}^{2}
$$

for all $f \in L^{2}(M)$, where the space $\ell^{2}\left(\mathbb{N}_{0}, \Sigma\right)$ and its norm are given, respectively, in (2.5) and (2.6).

We can interpret $\mathcal{F}=\mathcal{F}_{M}$ as saying that the Fourier transform is an isometry from $L^{2}(M)$ into $\ell^{2}\left(\mathbb{N}_{0}, \Sigma\right)$. The inverse of this Fourier transform can be then expressed by

$$
\left(\mathcal{F}^{-1} h\right)(x)=\sum_{j=0}^{\infty} \sum_{k=1}^{d_{j}} h(j, k) e_{j}^{k}(x)
$$

If $f \in L^{2}(M)$, we also write

$$
\widehat{f}(j)=\left(\begin{array}{c}
\widehat{f}(j, 1) \\
\vdots \\
\widehat{f}\left(j, d_{j}\right)
\end{array}\right) \in \mathbb{C}^{d_{j}},
$$

thus expressing the Fourier transform always as a column vector. In particular, we interpret $\widehat{e}_{j}^{k}(\ell)=\left(\widehat{e_{j}^{k}}(\ell, m)\right)_{m=1}^{d_{\ell}}$ as of a column and notice that

$$
\widehat{e_{j}^{k}}(\ell, m)=\delta_{j \ell} \delta_{k m}
$$

Smooth functions on $M$ can be characterised by

$$
\begin{aligned}
f \in C^{\infty}(M) & \Longleftrightarrow \forall N \exists C_{N}:|\widehat{f}(j, k)| \leq C_{N}\left(1+\lambda_{j}\right)^{-N} \text { for all } j, k \\
& \Longleftrightarrow \forall N \exists C_{N}:|\widehat{f}(j)| \leq C_{N}\left(1+\lambda_{j}\right)^{-N} \text { for all } j,
\end{aligned}
$$

where $|\widehat{f}(j)|$ is the norm of the vector $\widehat{f}(j) \in \mathbb{C}^{d_{j}}$. The implication " $\Leftarrow$ " here is immediate, while the implication " $\Rightarrow$ " follows from the Plancherel formula (2.4) and the fact that for $f \in C^{\infty}(M)$, we have $(I+E)^{N} f \in L^{2}(M)$ for all $N$. 
For $u \in \mathcal{D}^{\prime}(M)$, we denote its Fourier coefficient by $\widehat{u}(j, k):=u\left(\overline{e_{j}^{k}}\right)$. By duality, the space of distributions can be characterised by

$$
f \in \mathcal{D}^{\prime}(M) \Longleftrightarrow \exists M \exists C:|\widehat{u}(j, k)| \leq C\left(1+\lambda_{j}\right)^{M} \text { for all } j, k .
$$

We denote by $H^{s}(M)$ the usual Sobolev space over $L^{2}$ on $M$. This space can be defined in local coordinates or, by the fact that $E \in \Psi_{+e}^{v}(M)$ is positive and elliptic with $v>0$, can be characterised by

$$
\begin{aligned}
f \in H^{s}(M) & \Longleftrightarrow(I+E)^{s / v} f \in L^{2}(M) \Longleftrightarrow\left\{\left(1+\lambda_{j}\right)^{s / v} \widehat{f}(j)\right\}_{j} \in \ell^{2}\left(\mathbb{N}_{0}, \Sigma\right) \\
& \Longleftrightarrow \sum_{j=0}^{\infty} \sum_{k=1}^{d_{j}}\left(1+\lambda_{j}\right)^{2 s / v}|\widehat{f}(j, k)|^{2}<\infty .
\end{aligned}
$$

the last equivalence following from the Plancherel formula (2.4). For the characterisation of analytic functions (on compact manifolds $M$ ) we refer to Seeley [See69].

\section{Invariant operators and symbols on compact manifolds}

We now discuss an application of a notion of an invariant operator and of its symbol from Theorem 2.1 in the case $\mathcal{H}=L^{2}(M)$ and $\mathcal{H}^{\infty}=C^{\infty}(M)$ and describe its basic properties. We consider operators $T$ densely defined on $L^{2}(M)$ and make a natural assumption that their domain contains $C^{\infty}(M)$. We also note that while in Theorem 2.2 it was assumed that the operator $E_{o}$ is bounded on $\mathcal{H}$, this is no longer the case for the operator $E$ here. Indeed, an elliptic pseudo-differential operator $E \in \Psi_{+e}^{v}(M)$ of order $v>0$ is not bounded on $L^{2}(M)$.

Moreover, we do not assume that $T$ extends to a bounded operator on $L^{2}(M)$ to obtain analogues of properties (D) and (E) in Section 2 , because this is too restrictive from the point of view of differential operators. Instead, we show that, in the present setting, it suffices to assume that $T$ extends to a continuous operator on $\mathcal{D}^{\prime}(M)$ to reach the same conclusions.

So we combine the statement of Theorem 2.1 and the necessary modification of Theorem 2.2 to the setting of Section 3 as follows. We also remark that condition (iv) of the following theorem provides a correct formulation for a missing assumption in [DR14a, Theorem 3.1,(iv)].

Theorem 4.1. Let $M$ be a closed manifold, and let $T: C^{\infty}(M) \rightarrow L^{2}(M)$ be a linear operator. Then the following conditions are equivalent:

(i) $T\left(H_{j}\right) \subset H_{j}$ for each $j \in \mathbb{N}_{0}$; 
(ii) $T E e_{j}^{k}=E T e_{j}^{k}$ for each $j \in \mathbb{N}_{0}$ and $1 \leq k \leq j$;

(iii) for each $\ell \in \mathbb{N}_{0}$, there exists a matrix $\sigma(\ell) \in \mathbb{C}^{d_{\ell} \times d_{\ell}}$ such that

$$
\widehat{T e_{j}^{k}}(\ell, m)=\sigma(\ell)_{m k} \delta_{j \ell}
$$

for all $e_{j}^{k}$.

If, in addition, the domain of $T^{*}$ contains $C^{\infty}(M)$, then for each $\ell \in \mathbb{N}_{0}$, then the above conditions are equivalent to the condition

(iv) there exists a matrix $\sigma(\ell) \in \mathbb{C}^{d_{\ell} \times d_{\ell}}$ such that $\widehat{T f}(\ell)=\sigma(\ell) \widehat{f}(\ell)$ for all $f \in C^{\infty}(M)$.

The matrices $\sigma(\ell)$ in (iii) and (iv) coincide.

If $T$ extends to a linear continuous operator $T: \mathcal{D}^{\prime}(M) \rightarrow \mathcal{D}^{\prime}(M)$, then the above conditions are equivalent to the following conditions:

(v) $T P_{j}=P_{j} T$ on $C^{\infty}(M)$ for each $j \in \mathbb{N}_{0}$.

(vi) $T E=E T$ on $L^{2}(M)$.

If any of the equivalent conditions (i)-(iv) of Theorem 4.1 is satisfied, we say that the operator $T: C^{\infty}(M) \rightarrow L^{2}(M)$ is invariant (or is a Fourier multiplier) relative to $E$. We also say that $T$ is $E$-invariant or is an $E$-multiplier. This recovers the notion of invariant operators given by Theorem 2.1, with respect to the partitions $H_{j}$ 's in 2.1 which are fixed, being the eigenspaces of $E$. When there is no risk of confusion, we refer to such kind of operators as just invariant operators or as multipliers. It is clear from (ii) that the operator $E$ itself or functions of $E$ defined by the functional calculus are invariant relative to $E$.

We note that the boundedness of $T$ on $L^{2}(M)$, needed for conditions (D) and (F) in Theorem 2.1 and in Theorem 2.2, is now replaced by the condition that $T$ is continuous on $\mathcal{D}^{\prime}(M)$, which explored the additional structure of $L^{2}(M)$ and allows application to differential operators.

We call $\sigma$ in Theorem 4.1 (iii) and (iv) the matrix symbol of $T$ or simply the symbol of $T$. It is an element of the space $\Sigma=\Sigma_{M}$ defined by

$$
\Sigma_{M}:=\left\{\sigma: \mathbb{N}_{0} \ni \ell \mapsto \sigma(\ell) \in \mathbb{C}^{d_{\ell} \times d_{\ell}}\right\}
$$

Since the expression for the symbol depends only on the basis $e_{j}^{k}$ and not on the operator $E$ itself, this notion coincides with the symbol defined in Theorem 2.1 .

Let us comment on several conditions in Theorem 4.1 in this setting. Conditions (V) and (vi) are stronger than conditions (ii)-(iv). On one hand, clearly (vi) encompasses (iii). On the other hand, as shown in the proof, condition (v) implies (i) without the additional hypothesis that $T$ is continuous on $\mathcal{D}^{\prime}(M)$. 
In analogy to the strong commutativity in (), if $T$ is continuous on $\mathcal{D}^{\prime}(M)$, so that all the conditions (ii)-(vi) are equivalent, we may say that $T$ is strongly invariant relative to $E$.

The expressions in vil make sense, as both sides are defined (and even continuous) on $\mathcal{D}^{\prime}(M)$.

It is known from the general theory of densily defined operators on Hilbert spaces that without additional assumptions, conditions (V) and (vi) are generally not equivalent; see, e.g., Reed and Simon [RS80, Section VIII.5]. If $T$ is a differential operator, the additional assumption of continuity on $\mathcal{D}^{\prime}(M)$ for parts (V) and (vi) is satisfied. In [GW73, Section 1, Definition 1] Greenfield and Wallach called a differential operator $D$ an $E$-invariant operator if $E D=D E$, which is our condition (vi). However, Theorem 4.1 describes more general operators and reformulates them in the form of Fourier multipliers that are explored in the sequel.

There are several useful classes of symbols, in particular, the moderate growth class

$$
\mathcal{S}^{\prime}(\Sigma):=\left\{\sigma \in \Sigma: \exists N, C \text { such that }\|\sigma(\ell)\|_{o p} \leq C\left(1+\lambda_{\ell}\right)^{N} \forall \ell \in \mathbb{N}_{0}\right\}
$$

where $\|\sigma(\ell)\|_{o p}=\|\sigma(\ell)\|_{\mathscr{L}\left(H_{\ell}\right)}$ denotes the matrix multiplication operator norm with respect to $\ell^{2}\left(\mathbb{C}^{d_{\ell}}\right)$.

In the case $M$ is a compact Lie group and $E$ is a Laplacian on $G$, left-invariant operators on $G$, i.e., operators commuting with the left action of $G$, are also invariant relative to $E$ in the sense of Theorem 4.1, this is shown in Proposition 6.1 after we investigate in Section 6 the relation between the symbol in Theorem 4.1 and matrix symbols of operators on compact Lie groups. However, we need an adaptation of the above construction, since the natural decomposition into $H_{j}$ 's in (2.1) may in general violate the condition (3.1).

As in Section 2, since the notion of the symbol depends only on the basis, $\sigma_{I}(j)=I_{d_{j}}$ for the identity operator $T=I$, where $I_{d_{j}} \in \mathbb{C}^{I_{d_{j}} \times I_{d_{j}}}$ is the identity matrix, and for an operator $T=F(E)$, when it is well-defined by the spectral calculus,

$$
\sigma_{F(E)}(j)=F\left(\lambda_{j}\right) I_{d_{j}}
$$

Proof of Theorem 4.1. Once the basis $e_{j}^{k}$ is fixed, the equivalence of (ii), (ii) and (iv) follows from the equivalence of (A), (B) and (C) in Theorem 2.1.

(iii) $\Rightarrow$ (i). We first note that both $E T$ and $T E$ are well-defined on $e_{j}^{k}$ : for the former, since $e_{j}^{k}$ is smooth, $T e_{j}^{k} \in L^{2}(M)$, and hence in $\mathcal{D}^{\prime}(M)$, where $E$ is well-defined as a pseudo-differential operator, while, for the latter, since 
$E e_{j}^{k}=\lambda_{j} e_{j}^{k} \in H_{j} \subset C^{\infty}(M)$, which implies that $E e_{j}^{k}$ is in the domain of $T$. The rest of the proof is identical to that of $(\mathrm{E}) \Rightarrow(\mathrm{A})$ of Theorem 2.2.

(ii) $\Rightarrow$ (iii). This is the same as $(\mathrm{A}) \Rightarrow$ (E) of Theorem 2.2.

(V) $\Rightarrow$ (ii). We take $f \in H_{j}$. Then $P_{j} f=f \in C^{\infty}(M)$, so that, by condition (V), we have $T f=T P_{j} f=P_{j} T f \in H_{j}$, implying (ii).

(ii) $\Rightarrow$ (v). We now assume that $T$ is continuous on $\mathcal{D}^{\prime}(M)$. First, we show that (ii) implies that $\left\langle T g, \overline{e_{j}^{k}}\right\rangle=0$ for all $g \in H_{j}^{\perp} \subset L^{2}(M)$, in the sense of distributions. We can write $g=\sum_{\ell \neq j} \sum_{k=1}^{d_{\ell}}\left(g, e_{\ell}^{k}\right) e_{\ell}^{k}$ with the convergence in $L^{2}(M)$. Hence $T g=$ $\sum_{\ell \neq j} \sum_{k=1}^{d_{\ell}}\left(g, e_{\ell}^{k}\right) T e_{\ell}^{k}$ with the convergence being in $\mathcal{D}^{\prime}(M)$. Since $T e_{\ell}^{k} \in H_{\ell} \subset H_{j}^{\perp}$ for $\ell \neq j$, we conclude that $T g$ is orthogonal to $H_{j}$.

Let now $f \in C^{\infty}(M)$. Writing $f=f_{1}+f_{2}$ with $f_{1}=P_{j} f$, so that $f_{1} \in H_{j}$ and $f_{2} \in H_{j}^{\perp}$ are smooth, and $P_{j} f_{2}=0$, we have

$$
P_{j} T f=P_{j} T f_{1}+P_{j} T f_{2}=T f_{1}=T P_{j} f,
$$

since the above property implies that $P_{j} T f_{2}=0$.

(vi) $\Rightarrow$ (iii). This is trivial.

(iii) $\Rightarrow$ vii). Assume that $T$ is continuous on $\mathcal{D}^{\prime}(M)$. Then let us write $S:=E \circ T, D:=T \circ E$ and let $f \in L^{2}(M)$. We can write $f=\sum_{j=0}^{\infty} \sum_{k=1}^{d_{j}}\left(f, e_{j}^{k}\right) e_{j}^{k}$ with the series convergent in $L^{2}(M)$. Since both $S$ and $D$ are continuous on $\mathcal{D}^{\prime}(M)$,

$$
S f=\lim _{N} \sum_{j=0}^{N} \sum_{k=1}^{d_{j}}\left(f, e_{j}^{k}\right) S e_{j}^{k}=\lim _{N} \sum_{j=0}^{N} \sum_{k=1}^{d_{j}}\left(f, e_{j}^{k}\right) D e_{j}^{k}=D f .
$$

The limit should be understood in $\mathcal{D}^{\prime}(M)$. Indeed, with $f_{N}=\sum_{j=0}^{N} \sum_{k=1}^{d_{j}}\left(f, e_{j}^{k}\right) e_{j}^{k}$, we have $f_{N} \rightarrow f$ in $L^{2}$, and hence also $f_{N} \rightarrow f$ in $\mathcal{D}^{\prime}(M)$, which implies that $S f_{N} \rightarrow S f$ and $D f_{N} \rightarrow D f$ in $\mathcal{D}^{\prime}(M)$.

We now discuss how invariant operators can be expressed in terms of their symbols.

Proposition 4.2. An invariant operator $T_{\sigma}$ associated to the symbol $\sigma$ can be written as

$$
T_{\sigma} f(x)=\sum_{\ell=0}^{\infty} \sum_{m=1}^{d_{\ell}}(\sigma(\ell) \widehat{f}(\ell))_{m} e_{\ell}^{m}(x)=\sum_{\ell=0}^{\infty}[\sigma(\ell) \widehat{f}(\ell)]^{\top} e_{\ell}(x),
$$

where $[\sigma(\ell) \widehat{f}(\ell)]$ denotes a column-vector, and $[\sigma(\ell) \widehat{f}(\ell)]^{\top} e_{\ell}(x)$ denotes multiplication (the scalar product) of the column-vector $[\sigma(\ell) \widehat{f}(\ell)]$ by the column-vector $e_{\ell}(x)=\left(e_{\ell}^{1}(x), \cdots, e_{\ell}^{m}(x)\right)^{\top}$. In particular,

$$
\left(T_{\sigma} e_{j}^{k}\right)(x)=\sum_{m=1}^{d_{j}} \sigma(j)_{m k} e_{j}^{m}(x) .
$$


Moreover, if $\sigma \in \mathcal{S}^{\prime}(\Sigma)$ and $f \in C^{\infty}(M)$, then the convergence in (4.5) is uniform.

Proof. Formula (4.5) follows from Theorem 4.1 (iv), with uniform convergence for $f \in C^{\infty}(M)$ in view of (4.3). Then, using (4.5) and (3.6), we can calculate

$$
\begin{aligned}
\left(T_{\sigma} e_{j}^{k}\right)(x) & =\sum_{\ell=0}^{\infty} \sum_{m=1}^{d_{\ell}}\left(\sigma(\ell) \widehat{e_{j}^{k}}(\ell)\right)_{m} e_{\ell}^{m}(x)=\sum_{\ell=0}^{\infty} \sum_{m=1}^{d_{\ell}}\left(\sum_{i=1}^{d_{\ell}}(\sigma(\ell))_{m i} \widehat{e_{j}^{k}}(\ell, i)\right) e_{\ell}^{m}(x) \\
& =\sum_{\ell=0}^{\infty} \sum_{m=1}^{d_{\ell}} \sum_{i=1}^{d_{\ell}}(\sigma(\ell))_{m i} \delta_{j \ell} \delta_{k i} e_{\ell}^{m}(x)=\sum_{m=1}^{d_{j}}(\sigma(j))_{m k} e_{j}^{m}(x),
\end{aligned}
$$

yielding (4.6).

Theorem 2.3, which characterises invariant operators bounded on $L^{2}(M)$, now becomes the following theorem.

Theorem 4.3. An invariant linear operator $T: C^{\infty}(M) \rightarrow L^{2}(M)$ extends to a bounded operator from $L^{2}(M)$ to $L^{2}(M)$ if and only if its symbol $\sigma$ satisfies

$$
\sup _{\ell \in \mathbb{N}_{0}}\|\sigma(\ell)\|_{o p}<\infty,
$$

where $\|\sigma(\ell)\|_{o p}=\|\sigma(\ell)\|_{\mathscr{L}\left(H_{\ell}\right)}$ is the matrix multiplication operator norm with respect to $H_{\ell} \simeq \ell^{2}\left(\mathbb{C}^{d_{\ell}}\right)$. Moreover,

$$
\|T\|_{\mathscr{L}\left(L^{2}(M)\right)}=\sup _{\ell \in \mathbb{N}_{0}}\|\sigma(\ell)\|_{o p} .
$$

Theorem 2.3 can be extended to Sobolev spaces. We use the multiplication property for Fourier multipliers, which is a direct consequence of Proposition 2.4

Proposition 4.4. Let $S, T: C^{\infty}(M) \rightarrow L^{2}(M)$ be invariant operators with respect to $E$ such that the domain of $S \circ T$ contains $C^{\infty}(M)$. Then the operator $S \circ T: C^{\infty}(M) \rightarrow L^{2}(M)$ is also invariant with respect to $E$. Moreover,

$$
\sigma_{S \circ T}=\sigma_{S} \sigma_{T},
$$

where $\sigma_{S}$ denotes the symbol of $S$ and $\sigma_{T}$ denotes the symbols of $T$ with respect to the same orthonormal basis, i.e., $\sigma_{S \circ T}(j)=\sigma_{S}(j) \sigma_{T}(j)$ for all $j \in \mathbb{N}_{0}$.

Recalling Sobolev spaces $H^{s}(M)$ in (3.8) we have the following corollary.

Corollary 4.5. Let an invariant linear operator $T: C^{\infty}(M) \rightarrow C^{\infty}(M)$ have symbol $\sigma_{T}$ for which there exist $C>0$ and $m \in \mathbb{R}$ such that

$$
\left\|\sigma_{T}(\ell)\right\|_{o p} \leq C\left(1+\lambda_{\ell}\right)^{m / v}
$$

for all $\ell \in \mathbb{N}_{0}$. Then $T$ extends to a bounded operator from $H^{s}(M)$ to $H^{s-m}(M)$ for every $s \in \mathbb{R}$. 
Proof. We note that by (3.8), the condition that $T: H^{s}(M) \rightarrow H^{s-m}(M)$ is bounded is equivalent to the condition that the operator

$$
S:=(I+E)^{\frac{s-m}{v}} \circ T \circ(I+E)^{-\frac{s}{v}}
$$

is bounded on $L^{2}(M)$. By Proposition 4.4 and the fact that the powers of $E$ are pseudo-differential operators with diagonal symbols (see (4.4)), we have

$$
\sigma_{S}(\ell)=\left(1+\lambda_{\ell}\right)^{-\frac{m}{v}} \sigma_{T}(\ell)
$$

But then $\left\|\sigma_{S}(\ell)\right\|_{o p} \leq C$ for all $\ell$ in view of the assumption on $\sigma_{T}$, so the statement follows from Theorem 4.3 .

\section{Schatten classes of operators on compact manifolds}

In this section, we apply the constructions in the previous section to determine the membership of operators in Schatten classes and then apply them to a particular family of operators on $L^{2}(M)$.

As a consequence of Theorem 2.5, we can now characterise invariant operators in Schatten classes on compact manifolds. We note that this characterisation does not assume any regularity of the kernel or of the symbol. Observing that the conditions for the membership in the Schatten classes depend only on the basis $e_{j}^{k}$ and not on the operator $E$, we immediately obtain the following theorem.

Theorem 5.1. Let $0<r<\infty$. An invariant operator $T: L^{2}(M) \rightarrow L^{2}(M)$ is in $S_{r}\left(L^{2}(M)\right)$ if and only if $\sum_{\ell=0}^{\infty}\left\|\sigma_{T}(\ell)\right\|_{S_{r}}^{r}<\infty$. Moreover,

$$
\|T\|_{S_{r}\left(L^{2}(M)\right)}^{r}=\sum_{\ell=0}^{\infty}\left\|\sigma_{T}(\ell)\right\|_{S_{r}}^{r} .
$$

If an invariant operator $T: L^{2}(M) \rightarrow L^{2}(M)$ is in the trace class $S_{1}\left(L^{2}(M)\right)$, then

$$
\operatorname{Tr}(T)=\sum_{\ell=0}^{\infty} \operatorname{Tr}\left(\sigma_{T}(\ell)\right) .
$$

Remark 5.2. In Section 6 we establish a relation between the symbol introduced in Theorem 4.1 and the corresponding symbol in the setting of compact Lie groups (cf. [RT10, RT13]). In particular, the characterisation above extends the one obtained in [DR13, Theorem 3.7].

We now apply Theorem 5.1 to a determination of which powers of $E$ belong to which Schatten classes. But first we record a useful relation between the sequences $\left\{\lambda_{j}\right\}$ and $\left\{d_{j}\right\}$ of eigenvalues of $E$ and their respective multiplicities. 
Proposition 5.3. Let $M$ be a closed manifold of dimension $n$, and let $E \in \Psi_{+e}^{v}(M)$, with $v>0$. Then there exists a constant $C>0$ such that

$$
d_{j} \leq C\left(1+\lambda_{j}\right)^{\frac{n}{v}}
$$

for all $j \geq 1$. Moreover,

$$
\sum_{j=1}^{\infty} d_{j}\left(1+\lambda_{j}\right)^{-q}<\infty \quad \text { if and only if } \quad q>\frac{n}{v} .
$$

Proof. Since $\left(1+\lambda_{j}\right)^{1 / v}$ are the eigenvalues of the first-order elliptic positive operator $(I+E)^{1 / v}$ with multiplicities $d_{j}$, the Weyl eigenvalue counting formula for the operator $(I+E)^{1 / v}$ gives $\sum_{j:\left(1+\lambda_{j}\right)^{1 / v} \leq \lambda} d_{j}=C_{0} \lambda^{n}+O\left(\lambda^{n-1}\right)$ as $\lambda \rightarrow \infty$. This implies $d_{j} \leq C\left(1+\lambda_{j}\right)^{n / v}$ for sufficiently large $\lambda_{j}$, implying the estimate (5.1).

To prove (5.2), let us set $T:=(I+E)^{-q / 2}$. Then the eigenvalues of $T$ are $\left(1+\lambda_{j}\right)^{-q / 2}$ with multiplicities $d_{j}$. This implies

$$
\sum_{j=0}^{\infty} d_{j}\left(1+\lambda_{j}\right)^{-q}=\|T\|_{S_{2}}^{2} \asymp\|K\|_{L^{2}(M \times M)}^{2} .
$$

By the functional calculus of pseudo-differential operators, $T \in \Psi^{-v q / 2}(M)$, and so the integral kernel $K(x, y)$ of $T$ is smooth for $x \neq y$, and identifying points with their local coordinates, near the diagonal $x=y$, we have $|K(x, y)| \leq C_{\alpha}|x-y|^{-\alpha}$ for all $\alpha>n-v q / 2$; see, e.g., [Dui11] or [RT10, Theorem 2.3.1]. Thus order is sharp with respect to the order of the operator. Therefore, $K \in L^{2}(M \times M)$ if and only if there exists $\alpha$ such that $n>2 \alpha>2 n-v q$. Together with (5.3) this implies (5.2).

Proposition 5.4. Let $M$ be a closed manifold of dimension $n$, and let $E \in \Psi_{+e}^{v}(M)$ be a positive elliptic pseudo-differential operator of order $v>0$. Let $0<p<\infty$. Then

$$
(I+E)^{-\frac{\alpha}{v}} \in S_{p}\left(L^{2}(M)\right) \text { if and only if } \alpha>\frac{n}{p} .
$$

Proof. The operator $(I+E)^{-\frac{\alpha}{v}}$ is positive definite, and its singular values are $\left(1+\lambda_{j}\right)^{-\alpha / v}$ with respective multiplicities $d_{j}$. Therefore,

$$
\left\|(I+E)^{-\frac{\alpha}{v}}\right\|_{S_{p}}^{p}=\sum_{j=0}^{\infty} d_{j}\left(1+\lambda_{j}\right)^{-\frac{\alpha p}{v}}
$$

which is finite if and only if $\alpha p>n$ by (5.2), implying the statement. 


\section{Relation to the setting of compact Lie groups}

In the recent work [DR13], the authors studied Schatten classes of operators on compact Lie groups. We now explore how the notion of the symbol from Theorem 4.1 corresponds to the matrix-valued symbols on compact Lie groups and how the results for Schatten classes correspond to each other when $M=G$ is a compact Lie group. In this and the following sections, we assume that all operators are continuous on $\mathcal{D}^{\prime}(G)$, so that their integral kernels are distributions.

We give two types of decompositions of $L^{2}(G)$ into $H_{j}$ 's as in 2.1. First we choose $H_{j}$ 's determined by unitary irreducible representations of $G$. However, in this case the condition (3.1) may fail. Consequently, to view this analysis as a special case of the construction on manifolds in Section 4 with condition (3.1), we group representations corresponding to the same eigenvalue of the Laplacian together, to form a coarser decomposition of $L^{2}(G)$ into a direct sum of finitedimensional subspaces. The example of this types of partitions is given in Remark2.6 in the case of the torus $\mathbb{T}^{n}$.

Now we recall some basic definitions. Let $G$ be a compact Lie group of dimension $n$, equipped with the normalised Haar measure. Let $\widehat{G}$ denote the set of equivalence classes of continuous irreducible unitary representations of $G$. Since $G$ is compact, the set $\widehat{G}$ is discrete. For $[\xi] \in \widehat{G}$, by choosing a basis in the representation space of $\xi$, we can view $\xi$ as a matrix-valued function $\xi: G \rightarrow \mathbb{C}^{d_{\xi} \times d_{\xi}}$, where $d_{\xi}$ is the dimension of the representation space of $\xi$. By the Peter-Weyl theorem, the collection $\left\{\sqrt{d_{\xi}} \xi_{i j}: 1 \leq i, j \leq d_{\xi}\right.$, [ $\left.\left.\xi\right] \in \widehat{G}\right\}$ is an orthonormal basis of $L^{2}(G)$. We define the group Fourier transform of $f \in L^{1}(G)$ at $\xi$ by

$$
\mathcal{F}_{G} f(\xi) \equiv \widehat{f}(\xi):=\int_{G} f(x) \xi(x)^{*} d x,
$$

where $d x$ is the normalised Haar measure on $G$. If $\xi$ is a matrix representation, we have $\widehat{f}(\xi) \in \mathbb{C}^{d_{\xi} \times d_{\xi}}$. We note that this Fourier transform is different from the one we considered on manifolds in (3.3), which produced vector-valued Fourier coefficients instead of the matrix-valued ones obtained in 6.1).

The Fourier inversion formula is a consequence of the Peter-Weyl theorem, so we have

$$
f(x)=\sum_{[\xi] \in \widehat{G}} d_{\xi} \operatorname{Tr}(\xi(x) \widehat{f}(\xi)) .
$$

For each $[\xi] \in \widehat{G}$, the matrix elements of $\xi$ are the eigenfunctions for the Laplacian $\mathcal{L}_{G}$ (or the Casimir element of the universal enveloping algebra), with 
the same eigenvalues, which we denote by $-\lambda_{[\xi]}^{2}$, so we have

$$
-\mathcal{L}_{G} \xi_{i j}(x)=\lambda_{[\xi]}^{2} \xi_{i j}(x)
$$

for all $1 \leq i, j \leq d_{\xi}$. For a thorough discussion of Laplacians on compact Lie groups, we refer to [Ste70].

The weight for measuring the decay or growth of Fourier coefficients in this setting is $\langle\xi\rangle:=\left(1+\lambda_{[\xi]}^{2}\right)^{1 / 2}$, the eigenvalues of the elliptic first-order pseudodifferential operator $\left(I-\mathcal{L}_{G}\right)^{1 / 2}$. Parseval's identity takes the form

$$
\|f\|_{L^{2}(G)}=\left(\sum_{[\xi] \in \widehat{G}} d_{\xi}\|\widehat{f}(\xi)\|_{\mathrm{HS}}^{2}\right)^{1 / 2},
$$

where $\|\widehat{f}(\xi)\|_{\mathrm{HS}}^{2}=\operatorname{Tr}\left(\widehat{f}(\xi) \widehat{f}(\xi)^{*}\right)$, which defines the norm on $\ell^{2}(\widehat{G})$.

We define matrix-valued symbol $\tau_{A}(x, \xi) \in \mathbb{C}^{d_{\xi} \times d_{\xi}}$ for a linear continuous operator $A$ from $C^{\infty}(G)$ to $\mathcal{D}^{\prime}(G)$ by

$$
\tau_{A}(x, \xi):=\xi(x)^{*}(A \xi)(x) \in \mathbb{C}^{d_{\xi} \times d_{\xi}} .
$$

Then we have (see [RT10], [RT13]) the global quantization

$$
A f(x)=\sum_{[\xi] \in \widehat{G}} d_{\xi} \operatorname{Tr}\left(\xi(x) \tau_{A}(x, \xi) \widehat{f}(\xi)\right)
$$

in the sense of distributions, and the sum is independent of the choice of a representation $\xi$ from each equivalence class $[\xi] \in \widehat{G}$. If $A$ is a linear continuous operator from $C^{\infty}(G)$ to $C^{\infty}(G)$, the series $(6.5)$ is absolutely convergent and can be interpreted in the pointwise sense. We also write $A=\operatorname{Op}\left(\tau_{A}\right)$ for the operator $A$ given by the formula (6.5). We refer to [RT10, RT13] for the consistent development of this quantization and the corresponding symbolic calculus.

In the case of a left-invariant operator $A$, its symbol $\tau_{A}$ is independent of $x$, and formula (6.4) reduces to

$$
\tau_{A}(\xi)=\xi(x)^{*}(A \xi)(x)=A \xi(e),
$$

where $e$ is the unit element of the group.

We can now establish a correspondence between the two frameworks; the one in this paper and the one given in [DR13]. In the setting of compact Lie groups, the unitary dual being discrete, we can enumerate the representations as $\xi_{j}$ for $0 \leq j<\infty$. We enumerate the indices $(i, \ell)$ of each matrix $\xi(x)$ following the lexicographical order

$$
\left((i, \ell) \leq\left(i^{\prime}, \ell^{\prime}\right) \text { if } i<i^{\prime} \text { or }\left(i=i^{\prime} \text { and } \ell \leq \ell^{\prime}\right)\right) .
$$


We then fix the orthonormal basis $\left\{e_{j}^{k}\right\}$, given by

$$
\left\{e_{j}^{k}\right\}_{1 \leq k \leq d_{j}}=\left\{\sqrt{d_{\xi_{j}}}\left(\xi_{j}\right)_{i \ell}\right\}_{1 \leq i, \ell \leq d_{\xi_{j}}},
$$

where $d_{j}=d_{\xi_{j}}^{2}$ and $k$ represents an entry of the matrix of the representation following the lexicographical order described above. Then we have the subspaces

$$
H_{j} \equiv H_{\left[\xi_{j}\right]}:=\operatorname{span}\left\{\left(\xi_{j}\right)_{i \ell}: 1 \leq i, \ell \leq d_{\xi_{j}}\right\} .
$$

On a compact Lie group $G$, we can consider $E$ to be a bi-invariant Laplacian; see Stein [Ste70] for a discussion of such operators. Then, in view of the PeterWeyl theorem, eigenfunctions of $E$ are the functions $\left\{e_{j}^{k}\right\}_{1 \leq k \leq d_{j}}$, with norm 1 in $L^{2}(G)$ with respect to the normalised Haar measure, and corresponding to the same eigenvalue $\lambda_{j}$. However, the condition (3.1) does not hold in general, since nonequivalent representations in $\widehat{G}$ may give the same eigenvalues of the Laplacian.

We now observe that there is also a correspondence between the vector-valued Fourier transform introduced in (3.3) and the matrix-valued Fourier transform defined in (6.1). This correspondence can be established by applying once more the lexicographical order to the matrix-valued Fourier transform 6.1).

In order to study such correspondence, we define a bijection from the set of indices of the matrix-symbol $\{1, \ldots, d\}^{2}, d \in \mathbb{N}$, onto the set of indices $\left\{1, \ldots, d^{2}\right\}$ and calculate its inverse. For $(j, k) \in\{1, \ldots, d\}^{2}$, we define

$$
\Gamma_{d}(j, k):=(j-1) d+k
$$

The function $\Gamma_{d}$ is surjective; indeed, if $t \in\left\{1, \ldots, d^{2}\right\}$, then $j$ can be obtained from $j=\left\lfloor\frac{t-1}{d}\right\rfloor+1$, where $\lfloor\cdot\rfloor$ denotes the function defined for $x \geq 0$ by $\lfloor x\rfloor=$ $\max \left\{y \in \mathbb{N}_{0}: y \leq x\right\}$.

For the term $k$, we observe that $j-1=\left\lfloor\frac{t-1}{d}\right\rfloor$, hence $k=t-\left\lfloor\frac{t-1}{d}\right\rfloor d$. Since we are dealing with finite sets with the same number of elements, the injectivity of $\Gamma$ follows.

We can now establish correspondences between the Fourier transforms on $G=$ $M$ for $M$ viewed both as compact manifold and as a compact Lie group. Taking into account (6.1) and (6.7), we obtain

$$
\left(\mathcal{F}_{M} f\right)(i, t)=\left(f, e_{i}^{t}\right)_{L^{2}}=\sqrt{d_{\xi_{i}}}\left(\left(\mathcal{F}_{G} f\right)\left(\xi_{i}\right)\right)_{\left(t-\left\lfloor(t-1) / d_{\xi_{i}}\right\rfloor d_{\xi_{i}},\left\lfloor(t-1)\left(d_{\xi_{i}}\right)\right\rfloor+1\right)}
$$

for $i \in \mathbb{N}_{0}, 1 \leq t \leq d_{i}=d_{\xi_{i}}^{2}$.

In the another direction, we have

$$
\left(\left(\mathcal{F}_{G} f\right)\left(\xi_{\ell}\right)\right)_{i, j}=\frac{1}{\sqrt{d_{\xi_{\ell}}}}\left(\mathcal{F}_{M} f\right)\left(\ell, \Gamma_{d_{\xi_{\ell}}}(j, i)\right)
$$


for $1 \leq i, j \leq d_{\xi}$.

For the sake of simplicity, we introduce the notation

$$
\psi(t, d):=\left\lfloor\frac{t-1}{d}\right\rfloor+1, \quad \phi(t, d):=t-\left\lfloor\frac{t-1}{d}\right\rfloor d,
$$

where $t \in\left\{1, \ldots, d^{2}\right\}$. Formula (6.9) then becomes

$$
\left(\mathcal{F}_{M} f\right)(\ell, m)=\sqrt{d_{\xi_{\ell}}}\left(\left(\mathcal{F}_{G} f\right)\left(\xi_{\ell}\right)_{\left(\phi\left(m, d_{\tilde{\xi}}\right), \psi\left(m, d_{\xi_{\ell}}\right)\right)} .\right.
$$

Also,

$$
e_{j}^{k}=\left(\sqrt{d_{\xi_{j}}} \xi_{j}\right)_{\left(\psi\left(k, d_{\xi_{j}}\right), \phi\left(k, d_{\xi_{j}}\right)\right)} \text {. }
$$

In the calculations below, we use the following basic relations for the Fourier transform on a compact Lie group $G$ :

$$
\left(\mathcal{F}_{G}\left(\eta_{r s}\right)(\eta)\right)_{i j}=\int_{G} \eta_{r s}(x) \overline{\eta_{j i}(x)} d x=\frac{1}{d_{\eta}} \delta_{(i, j),(s, r)},
$$

which means that $\mathcal{F}_{G}\left(\eta_{r s}\right)(\eta)$ is the matrix of dimension $d_{\eta} \times d_{\eta}$ with the only entry different from $0,1 / d_{\eta}$ in the position $(s, r)$. We denote this matrix by $\frac{1}{d_{\eta}}\left(\delta_{(i, j),(s, r)}\right)_{i j}$. We have also

$$
\delta_{(i, j),(s, r)}= \begin{cases}1 & \text { if } i=s \text { and } r=j, \\ 0 & \text { otherwise. }\end{cases}
$$

Thus, for an invariant operator, we obtain

$$
\left(\mathcal{F}_{G}\left(T\left(\xi_{r s}\right)\right)\right)(\xi)=\tau(\xi)\left(\mathcal{F}_{G}\left(\xi_{r s}\right)(\xi)\right)=\tau(\xi) \frac{1}{d_{\xi}}\left(\delta_{(i, j),(s, r)}\right)_{i j} .
$$

In other words, $\left(\mathcal{F}_{G}\left(T\left(\xi_{r s}\right)\right)\right)(\xi)$ is a matrix of dimension $d_{\xi} \times d_{\xi}$, all of whose columms equal 0 except for the $r$-column, which equals the $s$-column of $\frac{1}{d_{\xi}} \tau(\xi)$.

We denote by $\sigma$ the symbol corresponding to $T$, and consider the orthonormal basis $\left\{e_{j}^{k}\right\}$ defined in (6.7) in the sense of (4.1) on manifolds. We denote the symbol introduced in (6.4) in the sense of groups by $\tau$. We now can find formulas relating the symbols $\tau$ and $\sigma$.

We begin by finding a formula for $\sigma$ in terms of $\tau$. By (6.11), (4.1) and (6.12), we obtain

$$
\begin{aligned}
\sigma(\ell)_{m i} & =\left(\mathcal{F}_{M}\left(T e_{\ell}^{i}\right)\right)(\ell, m)=\sqrt{d_{\xi_{\ell}}}\left(\left(\mathcal{F}_{G}\left(T e_{\ell}^{i}\right)\right)\left(\xi_{\ell}\right)\right)_{\left(\phi\left(m, d_{\xi_{\ell}}\right), \psi\left(m, d_{\xi_{\ell}}\right)\right)} \\
& \left.=\sqrt{d_{\xi_{\ell}}}\left(\left(\mathcal{F}_{G}\left(T\left(\sqrt{d_{\xi_{\ell}}} \xi_{\ell}\right)_{\psi\left(i, d_{\xi_{\ell}}\right.}\right), \phi\left(i, d_{\xi_{\ell}}\right)\right)\right)\left(\xi_{\ell}\right)\right)_{\left(\phi\left(m, d_{\xi_{\ell}}\right), \psi\left(m, d_{\xi_{\ell}}\right)\right)} \\
& =d_{\xi_{\ell}}\left(\left(\mathcal{F}_{G}\left(T\left(\xi_{\ell}\right)_{\psi\left(i, d_{\xi_{\ell}}\right), \phi\left(i, d_{\xi_{\ell}}\right)}\right)\right)\left(\xi_{\ell}\right)\right)_{\left(\phi\left(m, d_{\xi_{\ell}}\right), \psi\left(m, d_{\xi_{\ell}}\right)\right)} \\
& =d_{\xi_{\ell}} d_{\xi_{\ell}}^{-1}\left(\tau\left(\xi_{\ell}\right)\left(\delta_{\left((p, q),\left(\phi\left(i, d_{\xi_{\ell}}\right), \psi\left(i, d_{\xi_{\ell}}\right)\right)\right.}\right)_{p q}\right)_{\left(\phi\left(m, d_{\xi_{\ell}}\right), \psi\left(m, d_{\xi_{\ell}}\right)\right)} \\
& =\tau\left(\xi_{\ell}\right)_{\left(\phi\left(m, d_{\xi_{\ell}}\right), \phi\left(i, d_{\xi_{\ell}}\right)\right)} \delta_{\psi\left(i, d_{\xi_{\ell}}\right), \psi\left(m, d_{\xi_{\ell}}\right)} .
\end{aligned}
$$


Therefore,

$$
\sigma(\ell)_{m i}= \begin{cases}\tau\left(\xi_{\ell}\right)_{\left(\phi\left(m, d_{\xi_{\ell}}\right), \phi\left(i, d_{\xi_{\ell}}\right)\right)} & \text { if } \psi\left(m, d_{\xi_{\ell}}\right)=\psi\left(i, d_{\xi_{\ell}}\right) \\ 0 & \text { otherwise }\end{cases}
$$

Both functions $\phi$ and $\psi$ are periodic with respect to the first parameters $i$ and $m$, implying a periodic structure in the "big" manifold-symbol $\sigma$ composed of some copies of the "small" group-symbol $\tau$. The matrix below givew a graphical description of the relations (6.13). In it, the entries of $\tau\left(\xi_{\ell}\right)$ are distributed inside the matrix-symbol $\sigma$ according to 6.13 and $d:=d_{\xi_{\ell}}$.

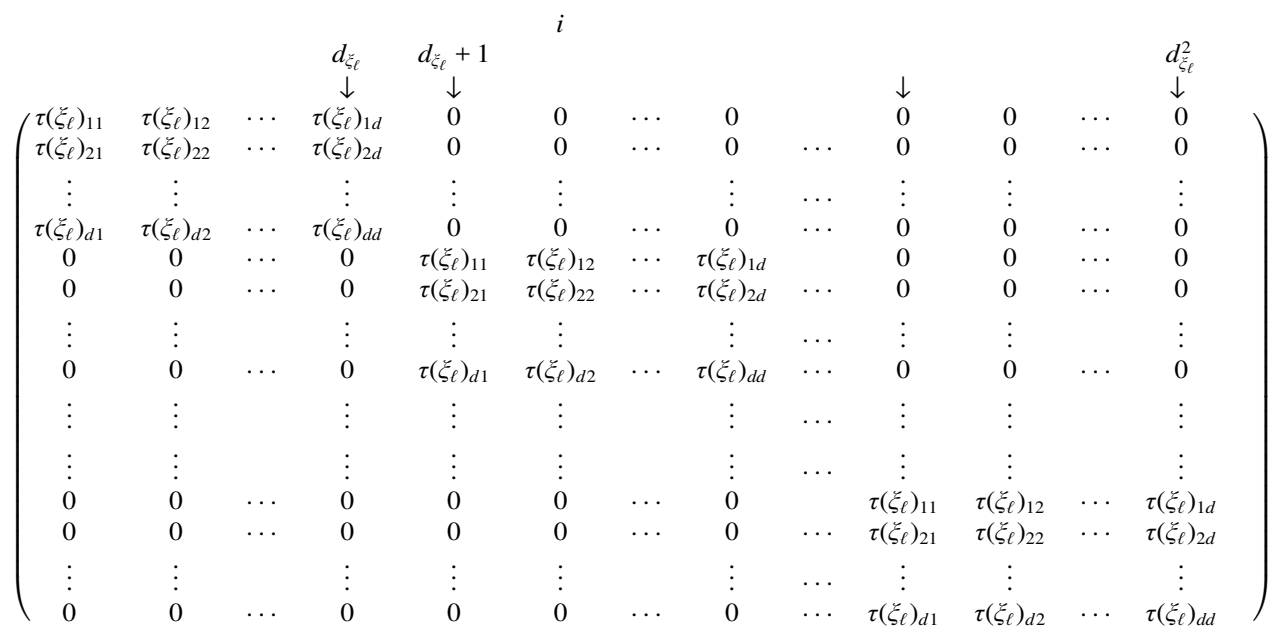

On the other hand, given the symbol $\sigma$, an application of equations (6.13) for $1 \leq m, i \leq d_{\xi_{\ell}}$ gives

$$
\tau\left(\xi_{\ell}\right)_{m i}=\sigma(\ell)_{m i}, \text { for } 1 \leq m, i \leq d_{\xi_{\ell}}
$$

Proposition 6.1 below shows that the Schatten quasi-norms $\|\cdot\|_{S_{r}}$ of the symbols $\tau$ and $\sigma$ are in agreement when $M=G$ is a compact Lie group. Thus, our results in Section 5] are an extension of those in [DR13] concerning Schatten classes. In particular, Theorem 5.1 extents [DR13, Theorem 3.7], as announced in Remark 5.2 .

We recall that on a compact Lie group $G$, we take $E$ to be a bi-invariant Laplacian.

Proposition 6.1. Let $G$ be a compact Lie group, $T: C^{\infty}(G) \rightarrow L^{2}(G)$ leftinvariant, and $\sigma$ be the symbol of $T$ in the sense of Theorem 2.1 and $\tau$ its symbol 
in the sense of groups as in (6.6). Then the these symbols are related by formulas (6.13)-(6.14). Furthermore, if $T$ continuous on $\mathcal{D}^{\prime}(G)$, then it also invariant relative to the family of $H_{j}$ 's as in (6.8) in the sense of Theorem 2.1 (in fact, it is also strongly invariant). Consequently, for every bounded left-invariant operator $T: L^{2}(G) \rightarrow L^{2}(G)$ and $0<r<\infty$,

$$
\|\sigma(\ell)\|_{S_{r}}^{r}=d_{\xi_{\ell}}\left\|\tau\left(\xi_{\ell}\right)\right\|_{S_{r}}^{r}
$$

and

$$
\sum_{\ell}\|\sigma(\ell)\|_{S_{r}}^{r}=\sum_{\ell} d_{\xi_{\ell}}\left\|\tau\left(\xi_{\ell}\right)\right\|_{S_{r}}^{r} .
$$

Proof. The invariance in the sense of groups as in (6.6) of the group-leftinvariant operators follows from the relation (6.13) between symbols and from the characterisation in Theorem 2.1

For the other statements, since $\|B\|_{S_{r}}=\||B|\|_{S_{r}}$ for Schatten quasi-norms, we can assume that $\sigma, \tau$ are symmetric, and hence diagonal. On the other hand, using the relation between $\sigma$ and $\tau$ in (6.13) and (6.14) and by examining the diagonal elements of $\sigma$ in (6.13), we obtain

$$
\|\sigma(\ell)\|_{S_{r}}^{r}=\sum_{m=1}^{d_{\xi_{\ell}}^{2}}\left|\sigma(\ell)_{m m}\right|^{r}=d_{\xi_{\ell}} \sum_{m=1}^{d_{\xi_{\ell}}}\left|\tau\left(\xi_{\ell}\right)_{m m}\right|^{r}=d_{\xi_{\ell}}\left\|\tau\left(\xi_{\ell}\right)\right\|_{S_{r}}^{r} .
$$

Thus $\|\sigma(\ell)\|_{S_{r}}^{r}=d_{\xi_{\ell}}\left\|\tau\left(\xi_{\ell}\right)\right\|_{S_{r}}^{r}$, and therefore, $\sum_{\ell}\|\sigma(\ell)\|_{S_{r}}^{r}=\sum_{\ell} d_{\xi_{\ell}}\left\|\tau\left(\xi_{\ell}\right)\right\|_{S_{r}}^{r}$.

We finish this section by describing an adaptation of the above construction, putting it in the framework of manifolds as described in Theorem 4.1. In the case of the torus $\mathbb{T}^{n}$, this is indicated in Remark 2.6. Recalling the definition of $H_{[\xi]}$ in (6.8) for each $[\xi] \in \widehat{G}$ and the notation $\lambda_{[\xi]}$ for the eigenvalues as in (6.3) for the sequence $0=\lambda_{0}^{2}<\lambda_{1}^{2}<\lambda_{2}^{2}<\ldots$ of eigenvalues of $-\mathcal{L}_{G}$ counted without multiplicities, we set

$$
\widetilde{H_{\ell}}:=\bigoplus_{\substack{\left.\left.[\xi] \in \widehat{G} \\ \lambda_{[\xi}\right]\right]_{\ell}}} H_{[\xi]}=\bigoplus_{\substack{[\xi] \in \widehat{G} \\ \lambda_{[\xi}\left[\lambda_{\ell}\right.}} \operatorname{span}\left\{\xi_{i k}: 1 \leq i, k \leq d_{\xi}\right\}, \quad \ell \in \mathbb{N}_{0} .
$$

The family of $\widetilde{H_{\ell}}$ 's is the collection of eigenspaces of the elliptic differential operator $\mathcal{L}_{G}$ for which the condition (3.1) is satisfied. The symbols $\sigma$ and $\widetilde{\sigma}$ of an invariant operator $T$ with respect to the partitions $H_{j}$ 's and $\widetilde{H}_{\ell}$ 's, respectively, are related by

$$
\widetilde{\sigma}(\ell)=\bigotimes_{\substack{\left[\xi_{j}\right] \in \widehat{G} \\ \lambda_{\left[\xi_{j}\right]}=\lambda_{\ell}}} \sigma(j)
$$


with $\widetilde{\sigma}(\ell) \in \mathbb{C}^{\widetilde{d}_{\ell}} \times \widetilde{d}_{\ell}$ and

$$
\widetilde{d}_{\ell}=\sum_{\substack{\left[\xi_{j}\right] \in \widehat{G} \\ \lambda_{\left[\xi_{j}\right]=\lambda_{\ell}}}} d_{j}=\sum_{\substack{\left[\tilde{\xi}_{j}\right] \in \widehat{G} \\ \lambda^{\lambda}\left[\xi_{j}\right]=\lambda_{\ell}}} d_{\xi_{j}}^{2}
$$

Recalling the relation (6.13) between the symbol $\sigma$ in the sense of Theorem 2.1 and the group symbol $\tau$ as in (6.6), given by

$$
\sigma(j) \equiv \sigma\left(\xi_{j}\right)=\left(\begin{array}{cccc}
\tau\left(\xi_{j}\right) & 0 & \cdots & 0 \\
0 & \tau\left(\xi_{j}\right) & \cdots & 0 \\
\vdots & \vdots & \cdots & \vdots \\
0 & 0 & \cdots & \tau\left(\xi_{j}\right)
\end{array}\right)
$$

we see that the formula (6.16) provides the further relation between the symbol $\tilde{\sigma}$ in the sense of manifolds (in Theorem 4.1) and the group symbol $\tau$. Therefore, if $\lambda_{\left[\xi_{1}\right]}=\ldots=\lambda_{\left[\xi_{m}\right]}=\lambda_{\ell}$ for non-equivalent representations $\left[\xi_{1}\right], \ldots,\left[\xi_{m}\right] \in \widehat{G}$, then

$$
\widetilde{\sigma}(\ell)=\left(\begin{array}{cccc}
\sigma\left(\xi_{1}\right) & 0 & \cdots & 0 \\
0 & \sigma\left(\xi_{2}\right) & \cdots & 0 \\
\vdots & \vdots & \cdots & \vdots \\
0 & 0 & \cdots & \sigma\left(\xi_{m}\right)
\end{array}\right)
$$

In particular, we obtain the following corollary.

Corollary 6.2. Let $G$ be a compact Lie group, and let $T: C^{\infty}(G) \rightarrow L^{2}(G)$ be a linear operator, continuous on $\mathcal{D}^{\prime}(G)$. If $T$ is left-invariant then it is also invariant relative to the operator $\mathcal{L}_{G}$ (in the sense of Theorem 4.1). The corresponding symbols are related by formulas 6.16-6.18).

\section{Kernels of invariant operators on compact manifolds}

In this section, we describe invariant operators relative to $E$ in terms of their kernels. We first observe that if $T=T_{\sigma}$ with symbol $\sigma$ is invariant, by expanding (4.5) 
we can write

$$
\begin{aligned}
T_{\sigma} f(x)= & \sum_{\ell=0}^{\infty} \sum_{m=1}^{d_{\ell}}(\sigma(\ell) \widehat{f}(\ell))_{m} e_{\ell}^{m}(x)=\sum_{\ell=0}^{\infty} \sum_{m=1}^{d_{\ell}} \sum_{k=1}^{d_{\ell}} \sigma(\ell)_{m k} \widehat{f}(\ell)_{k} e_{\ell}^{m}(x) \\
& =\sum_{\ell=0}^{\infty} \sum_{m=1}^{d_{\ell}} \sum_{k=1}^{d_{\ell}} \sigma(\ell)_{m k} e_{\ell}^{m}(x) \int_{M} f(y) \overline{e_{\ell}^{k}(y)} d y \\
& =\int_{M}\left(\sum_{\ell=0}^{\infty} \sum_{m=1}^{d_{\ell}} \sum_{k=1}^{d_{\ell}} \sigma(\ell)_{m k} e_{\ell}^{m}(x) \overline{e_{\ell}^{k}(y)}\right) f(y) d y .
\end{aligned}
$$

Hence, the integral kernel $K(x, y)$ of $T_{\sigma}$ is given by

$$
K(x, y)=\sum_{\ell=0}^{\infty} \sum_{m=1}^{d_{\ell}} \sum_{k=1}^{d_{\ell}} \sigma(\ell)_{m k} e_{\ell}^{m}(x) \overline{e_{\ell}^{k}(y)}
$$

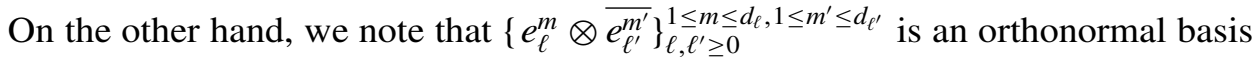
of $L^{2}(M \times M)$. If $T$ is Hilbert-Schmidt on $L^{2}(M)$, not necessarily invariant, then its kernel $K$ is square-integrable, and we can write its decomposition in this basis as

$$
K(x, y)=\sum_{\ell=0}^{\infty} \sum_{\ell^{\prime}=0}^{\infty} \sum_{m=1}^{d_{\ell}} \sum_{m^{\prime}=1}^{d_{\ell^{\prime}}}\left(\left(\mathcal{F}_{M} \otimes \overline{\mathcal{F}_{M}}\right) K\right)\left(\ell, m, \ell^{\prime}, m^{\prime}\right) e_{\ell}^{m}(x) \overline{e_{\ell^{\prime}}^{m^{\prime}}(y)}
$$

where $\left(\left(\mathcal{F}_{M} \otimes \overline{\mathcal{F}_{M}}\right) K\right)\left(\ell, m, \ell^{\prime}, m^{\prime}\right)$ denote the Fourier coefficients of $K$ with respect to the basis $\left\{e_{\ell}^{m} \otimes \overline{e_{\ell^{\prime}}^{m^{\prime}}}\right\}$ given by

$$
\begin{aligned}
\left(\left(\mathcal{F}_{M} \otimes \overline{\mathcal{F}_{M}}\right) K\right)\left(\ell, m, \ell^{\prime}, m^{\prime}\right) & =\left(K, e_{\ell}^{m}(x) \overline{e_{\ell^{\prime}}^{m^{\prime}}(y)}\right)_{L^{2}(M \times M)} \\
& =\int_{M \times M} K(x, y) \overline{e_{\ell}^{m}(x)} e_{\ell^{\prime}}^{m^{\prime}}(y) d x d y .
\end{aligned}
$$

We observe from (7.1) and (7.2) that $T$ is invariant relative to $\left(E,\left\{e_{\ell}^{m}\right\}_{\ell \geq 0}^{1 \leq m \leq d_{\ell}}\right)$ if and only if

$$
\left(\left(\mathcal{F}_{M} \otimes \overline{\mathcal{F}_{M}}\right) K\right)\left(\ell, m, \ell^{\prime}, m^{\prime}\right)= \begin{cases}\sigma(\ell)_{m m^{\prime}} & \text { if } \ell=\ell^{\prime} \\ 0 & \text { otherwise }\end{cases}
$$


For example, from (7.1), we obtain

$$
\begin{aligned}
\left(K, e_{\ell}^{m}(x) \overline{e_{\ell^{\prime}}^{m^{\prime}}(y)}\right)_{L^{2}(M \times M)} & =\int_{M \times M}\left(\sum_{j=0}^{\infty} \sum_{k=1}^{d_{j}} \sum_{i=1}^{d_{j}} \sigma(j)_{k i} e_{j}^{k}(x) \overline{e_{j}^{i}(y)}\right) \overline{e_{\ell}^{m}(x)} e_{\ell^{\prime}}^{m^{\prime}}(y) d x d y \\
& =\sum_{j=0}^{\infty} \sum_{k=1}^{d_{j}} \sum_{i=1}^{d_{j}} \sigma(j)_{k i} \int_{M} e_{j}^{k}(x) \overline{e_{\ell}^{m}(x)} d x \int_{M} e_{\ell^{\prime}}^{m^{\prime}}(y) \overline{e_{j}^{i}(y)} d y \\
& = \begin{cases}\sigma(\ell)_{m m^{\prime}} & \text { if } \ell=\ell^{\prime}, \\
0 & \text { otherwise. }\end{cases}
\end{aligned}
$$

We now introduce some notation which is useful for defining a suitable setting to study the above Fourier coefficients and the relation between an operator's kernel and symbol. Let

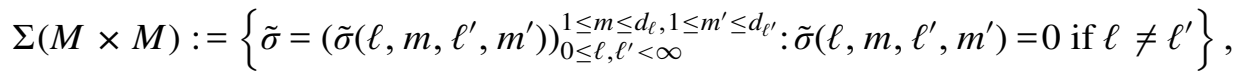

$$
\begin{aligned}
& \mathcal{K}:=\left\{K \in \mathcal{D}^{\prime}(M \times M): K \text { defines an invariant operator relative to } E\right\} \text {. }
\end{aligned}
$$

We now consider the mapping $K \mapsto\left(\mathcal{F}_{M} \otimes \overline{\mathcal{F}_{M}}\right) K$ from $\mathcal{K}$ into $\Sigma(M \times M)$. We can identify the family of symbols $\Sigma(M \times M)$ with the matrices $\bigcup_{\ell} \mathbb{C}^{d_{\ell} \times d_{\ell}}$ by letting $\tilde{\sigma} \equiv \sigma$ be such that $\sigma(\ell)_{m m^{\prime}}=\tilde{\sigma}\left(\ell, m, \ell, m^{\prime}\right)$. In this way, we also get the identification $\Sigma(M \times M) \simeq \Sigma_{M}=\Sigma$ with $\Sigma$ from (4.2).

For $1 \leq p<\infty$, we define

$$
\ell^{p}(\Sigma)=\left\{\sigma \in \Sigma: \sum_{\ell=0}^{\infty}\|\sigma(\ell)\|_{S_{p}}^{p}<\infty\right\} .
$$

On $\ell^{p}(\Sigma)$, we define the norm

$$
\|\sigma\|_{\ell^{p}(\Sigma)}:=\left(\sum_{\ell=0}^{\infty}\|\sigma(\ell)\|_{S_{p}}^{p}\right)^{1 / p}, \quad 1 \leq p<\infty .
$$

For $p=\infty$, we define $\ell^{\infty}(\Sigma)=\left\{\sigma \in \Sigma: \sup _{\ell \in \mathbb{N}_{0}}\|\sigma(\ell)\|_{o p}<\infty\right\}$, and we endow $\ell^{\infty}(\Sigma)$ with the norm $\|\sigma\|_{\ell^{\infty}(\Sigma)}:=\sup _{\ell \in \mathbb{N}_{0}}\|\sigma(\ell)\|_{o p}$. We still sometimes denote the integral operator with kernel $K$ by $T_{K}$.

In terms of the norms $\ell^{p}(\Sigma)$, for invariant operators, Theorem 4.3 can be formulated as

$$
T \in \mathscr{L}\left(L^{2}(M)\right) \Longleftrightarrow \sigma_{T} \in \ell^{\infty}(\Sigma),
$$

and Theorem 5.1 can be formulated as

$$
T \in S_{p}\left(L^{2}(M)\right) \Longleftrightarrow \sigma_{T} \in \ell^{p}(\Sigma)
$$


for $0<p<\infty$.

For the formulation of the following theorem, we use the mixed-norm $L^{p}$ spaces $L_{x}^{p_{1}} L_{y}^{p_{2}}$ on the manifold $M$ for $1 \leq p_{1}, p_{2} \leq \infty$. A measurable function $K(x, y)$ is said to belong to $L_{x}^{p_{1}} L_{y}^{p_{2}}(M \times M)$ if \|\|$K(x, y)\left\|_{L_{y}^{p_{2}}}\right\|_{L_{x}^{p_{1}}}<\infty$. On $L_{x}^{p_{1}} L_{y}^{p_{2}}(M \times M)$, we consider the norm $\|\cdot\|_{L_{x}^{p_{1}} L_{y}^{p_{2}}}:=\|\| \cdot\left\|_{L_{y}^{p_{y}}}\right\|_{L_{x}^{p_{1}}}$. We also define

$$
L^{\left(p_{1}, p_{2}\right)}(M \times M):=L_{x}^{p_{1}} L_{y}^{p_{2}}(M \times M) \cap L_{y}^{p_{1}} L_{x}^{p_{2}}(M \times M),
$$

and endow $L^{\left(p_{1}, p_{2}\right)}(M \times M)$ with norm $\|\cdot\|_{L^{\left(p_{1}, p_{2}\right)}}:=\max \left\{\|\cdot\|_{L_{x}^{p_{1}} L_{y}^{p_{2}}},\|\cdot\|_{L_{y}^{p_{1}} L_{x}^{p_{2}}}\right\}$ In general, $L^{\left(p_{1}, p_{2}\right)} \neq L^{\left(p_{2}, p_{1}\right)}$.

The basic properties of mixed-norm $L^{p}$ spaces for many variables were first studied by Benedek and Panzone in [BP61]. In particular, they proved a version of Stein's interpolation of operators theorem and, as a consequence, the Riesz-Thorin theorem in that setting. A slight modification allows us to apply the Riesz-Thorin theorem when the operator $T$ acts from a mixed-norm $L^{p}$ space to an $\ell^{p}(\Sigma)$-space.

Theorem 7.1. Let $1 \leq p \leq 2$ and $K \in \mathcal{K} \cap L^{\left(p^{\prime}, p\right)}$. Then $\left(\mathcal{F}_{M} \otimes \overline{\mathcal{F}_{M}}\right) K \in \ell^{p^{\prime}}(\Sigma)$, where $\frac{1}{p}+\frac{1}{p^{\prime}}=1$.

Proof. If $p=2$, then $p^{\prime}=2$. From $K \in \mathcal{K} \cap L_{x}^{2} L_{y}^{2} \cap L_{y}^{2} L_{x}^{2}=\mathcal{K} \cap L_{x, y}^{2} \subset L_{x, y}^{2}$, we get a Hilbert-Schmidt operator $T_{K}$. On the other hand, by Theorem 5.1 with $r=2$, we get $\sum_{\ell}\|\sigma(\ell)\|_{S_{2}}^{2}<\infty$, where $\sigma$ is the symbol of $T_{K}$. Hence, by (7.3), we obtain $\left(\mathcal{F}_{M} \otimes \overline{\mathcal{F}_{M}}\right) K \in \ell^{2}(\Sigma)$.

If $p=1$, then $p^{\prime}=\infty$. If $K \in \mathcal{K} \cap L_{x}^{\infty} L_{y}^{1} \cap L_{y}^{\infty} L_{x}^{1}$, by Schur's Lemma we get $T_{K} \in \mathscr{L}\left(L^{r}(M)\right)$ for all $1 \leq r \leq \infty$. In particular, $T_{K} \in \mathscr{L}\left(L^{2}(M)\right)$, and, by Theorem 4.3, the symbol $\sigma$ of $T_{K}$ satisfies $\sup _{\ell}\|\sigma(\ell)\|_{o p}<\infty$. By (7.3),

$$
\left\|\left(\mathcal{F}_{M} \otimes \overline{\mathcal{F}_{M}}\right) K\right\|_{\ell^{\infty}(\Sigma)}=\sup _{\ell}\|\sigma(\ell)\|_{o p} .
$$

Hence $\left(\mathcal{F}_{M} \otimes \overline{\mathcal{F}_{M}}\right) K \in \ell^{\infty}(\Sigma)$.

We have shown that

$$
\left(\mathcal{F}_{M} \otimes \overline{\mathcal{F}_{M}}\right): \mathcal{K} \cap L^{(2,2)} \longrightarrow \ell^{2}(\Sigma)
$$

and

$$
\left(\mathcal{F}_{M} \otimes \overline{\mathcal{F}_{M}}\right): \mathcal{K} \cap L^{(\infty, 1)} \longrightarrow \ell^{\infty}(\Sigma) .
$$

By the Riesz-Thorin interpolation theorem between $L^{(r, s)}$ and $\ell^{p}(\Sigma)$ spaces (see [BP61, Theorem 2]), we obtain

$$
\left(\mathcal{F}_{M} \otimes \overline{\mathcal{F}_{M}}\right): \mathcal{K} \cap L^{\left(p_{1}, p_{2}\right)} \longrightarrow \ell^{q}(\Sigma),
$$


with

$$
\frac{1}{p_{1}}=\frac{1-\theta}{2}+\frac{\theta}{\infty}, \quad \frac{1}{p_{2}}=\frac{1-\theta}{2}+\frac{\theta}{1}, \quad \frac{1}{q}=\frac{1-\theta}{2}+\frac{\theta}{\infty}
$$

for $0 \leq \theta \leq 1$. Hence

$$
p_{1}=\frac{2}{1-\theta}, p_{2}=\frac{2}{1+\theta}, q=\frac{2}{1-\theta} .
$$

If $p=\frac{2}{1+\theta}$, then $\theta=\frac{2-p}{p}$ and $\frac{2}{1-\theta}=\frac{p}{p-1}=p^{\prime}$. Thus

$$
\left(\mathcal{F}_{M} \otimes \overline{\mathcal{F}_{M}}\right): \mathcal{K} \cap L^{\left(p^{\prime}, p\right)} \longrightarrow \ell^{p^{\prime}}(\Sigma),
$$

which completes the proof.

The following corollary is an immediate consequence of Theorems 7.1 and 5.1 It furnishes a sufficient kernel condition for Schatten classes with index $p^{\prime} \geq 2$.

Corollary 7.2. If $1 \leq p \leq 2$ and $K \in \mathcal{K} \cap L^{\left(p^{\prime}, p\right)}(M \times M)$ then $T_{K} \in$ $S_{p^{\prime}}\left(L^{2}(M)\right)$.

We recall that sufficient conditions of the type above in terms of kernels are not possible for $0<p^{\prime}<2$, as a consequence of a Carleman's example. Corollary 7.2 is known for general integral operators (cf. [Rus74, Theorem 3]). Here we have deduced a particular version for invariant operators with a simple proof by applying the notion of symbol.

We now describe another representation of the kernel as the "generalised" Fourier transform of the symbol. From formula (7.1), we have

$$
\begin{aligned}
K(x, y) & =\sum_{\ell=0}^{\infty} \sum_{m=1}^{d_{\ell}} \sum_{k=1}^{d_{\ell}} \sigma(\ell)_{m k} e_{\ell}^{m}(x) \overline{e_{\ell}^{k}(y)}=\sum_{\ell=0}^{\infty} \operatorname{Tr}\left(e_{\ell}(x)^{\top} \sigma(\ell) \overline{e_{\ell}(y)}\right) \\
& =\sum_{\ell=0}^{\infty} \operatorname{Tr}\left(\sigma(\ell) \overline{e_{\ell}(y)} e_{\ell}(x)^{\top}\right)=\sum_{\ell=0}^{\infty} \operatorname{Tr}\left(\sigma(\ell) Q_{\ell}(x, y)\right),
\end{aligned}
$$

where $Q_{\ell}(x, y)=\overline{e_{\ell}(y)} e_{\ell}(x)^{\top} \in \mathbb{C}^{d_{\ell} \times d_{\ell}}$.

We notice that the matrix-valued function $\left(Q_{\ell}(x, y)\right)_{m k}=\overline{e_{\ell}^{m}(x)} e_{\ell}^{k}(y)$ is of rank 1 for every $\ell$. Indeed, $\left(Q_{\ell}(x, y)\right)_{m k}$ is nothing other the tensor product of the vectors $e_{\ell}(x), \overline{e_{\ell}(y)} \in \mathbb{C}^{d_{\ell}}$. Since on a normed space $F,\|u \otimes v\|_{o p}=\|u\|_{F}\|v\|_{F}$, we get

$$
\left\|Q_{\ell}(x, y)\right\|_{o p}=\left\|e_{\ell}(x)\right\|_{\ell^{2}\left(\mathbb{C}^{\left.d_{\ell}\right)}\right.}\left\|e_{\ell}(y)\right\|_{\ell^{2}\left(\mathbb{C}^{d_{\ell}}\right)} .
$$

Вy (7.2),

$$
\sigma(\ell)=\int_{M \times M} K(x, y) Q_{\ell}(x, y)^{*} d x d y
$$


Hence

$$
\begin{aligned}
\|\sigma(\ell)\|_{o p} & \leq\|K\|_{L^{1}(M \times M)} \sup _{x, y}\left\|Q_{\ell}(x, y)^{*}\right\|_{o p} \\
& =\|K\|_{L^{1}(M \times M)} \sup _{x, y}\left\|e_{\ell}(x)\right\|_{\ell^{2}\left(\mathbb{C}^{d_{\ell}}\right)}\left\|e_{\ell}(y)\right\|_{\ell^{2}\left(\mathbb{C}^{d_{\ell}}\right)} .
\end{aligned}
$$

Remark 7.3. The condition $K \in L^{1}(M \times M)$ alone does not guarantee the $L^{2}$ boundedness of the corresponding integral operator $T$. Indeed, consider $M=\mathbb{T}^{1}$, $g \in L^{1}\left(\mathbb{T}^{1}\right) \backslash L^{2}\left(\mathbb{T}^{1}\right), h \equiv 1 \in L^{1}\left(\mathbb{T}^{1}\right)$, and the kernel

$$
K(\theta, \phi):=g(\theta) h(\phi) \in L^{1}\left(\mathbb{T}^{1} \times \mathbb{T}^{1}\right) .
$$

It is easy to see that the kernel $K(\theta, \phi)$ does not define an operator from $L^{2}\left(\mathbb{T}^{1}\right)$ into $L^{2}\left(\mathbb{T}^{1}\right)$. For example, with $f=1 \in L^{2}\left(\mathbb{T}^{1}\right)$, we have

$$
(T 1)(\theta)=g(\theta) \int_{\mathbb{T}^{1}} h(\phi) d \phi=g(\theta) \notin L^{2}\left(\mathbb{T}^{1}\right) .
$$

\section{Applications to the nuclearity of operators in $L^{p}(M)$}

We now turn to the study of nuclearity in $L^{p}$-spaces on closed manifolds. Sufficient conditions for $r$-nuclearity on $L^{p}$ on compact Lie groups have been established in [DR14b]. The study of nuclearity on $L^{p}$ in this section relies on the analysis of suitable kernel decompositions and the relation between kernels and symbols described in Section 7

Let $E$ and $F$ be Banach spaces and $0<r \leq 1$. A linear operator $T$ from $E$ into $F$ is called $r$-nuclear if there exist sequences $\left\{x_{n}^{\prime}\right\}$ in $E^{\prime}$ and $\left\{y_{n}\right\}$ in $F$ such that

$$
. T x=\sum_{n}\left\langle x, x_{n}^{\prime}\right\rangle y_{n} \text { and } \sum_{n}\left\|x_{n}^{\prime}\right\|_{E^{\prime}}^{r}\left\|y_{n}\right\|_{F}^{r}<\infty \text {. }
$$

1-nuclear operators are known as nuclear operators. In that case, this definition agrees with the concept of trace class operator in the setting of Hilbert spaces $(E=F=H)$. More generally, Oloff proved in [Olo72] that the class of $r$ nuclear operators coincides with the Schatten class $S_{r}(H)$ when $E=F=H$ and $0<r \leq 1$.

The concept of $r$-nuclearity was introduced by Grothendieck [Gro55], and has application to questions of the distribution of eigenvalues of operators in Banach spaces via, e.g., the Grothendieck-Lidskii formula. We refer to [DR14b] for several conclusions in the setting of compact Lie groups concerning summability and distribution of eigenvalues of operators on $L^{p}$-spaces using information on their $r$-nuclearity. Since these arguments are then purely functional analytic, they apply 
equally well in the present setting of closed manifolds; hence we omit the arguments but instead refer the reader to [DR14b] for several relevant applications.

The $r$-nuclear operators on Lebesgue spaces are characterised by the following theorem; cf. [Del10]. In the statement of it, $\left(\Omega_{1}, \mathcal{M}_{1}, \mu_{1}\right)$ and $\left(\Omega_{2}, \mathcal{M}_{2}, \mu_{2}\right)$ are $\sigma$-finite measure spaces.

Theorem 8.1. Let $1 \leq p_{1}, p_{2}<\infty, 0<r \leq 1$, and $q_{1}$ be such that $\frac{1}{p_{1}}+\frac{1}{q_{1}}=1$. An operator $T: L^{p_{1}}\left(\mu_{1}\right) \rightarrow L^{p_{2}}\left(\mu_{2}\right)$ is $r$-nuclear if and only if there exist sequences $\left\{g_{n}\right\}_{n}$ in $L^{p_{2}}\left(\mu_{2}\right)$, and $\left\{h_{n}\right\}_{n}$ in $L^{q_{1}}\left(\mu_{1}\right)$ such that

$$
\sum_{n=1}^{\infty}\left\|g_{n}\right\|_{L^{p_{2}}}^{r}\left\|h_{n}\right\|_{L^{q_{1}}}^{r}<\infty
$$

and

$$
T f(x)=\int\left(\sum_{n=1}^{\infty} g_{n}(x) h_{n}(y)\right) f(y) d \mu_{1}(y), \quad \text { for a.e. } x
$$

for all $f \in L^{p_{1}}\left(\mu_{1}\right)$.

In order to study nuclearity on $L^{p}(M)$ spaces for a given compact manifold $M$ of dimension $n$, we introduce a function $\Lambda(j, k ; n, p)$ which controls the $L^{p}$ norms of the family of eigenfunctions $\left\{e_{j}^{k}\right\}$ of the operator $E$, i.e., we suppose that $\Lambda(j, k ; n, p)$ is such that

$$
\left\|e_{j}^{k}\right\|_{L^{p}(M)} \leq \Lambda(j, k ; n, p)
$$

In particular, if $\Lambda$ is such a function, then

$$
\left\|e_{j}^{k}\right\|_{L^{p}(M)} \leq \operatorname{vol}(M)^{1 / p} \Lambda(j, k ; n, \infty) .
$$

When $M=G$ is a compact Lie group, efficient $\left\|e_{j}^{k}\right\|_{L^{p}(G)}$ bounds can be obtained; cf. [DR14b]. The estimation of $L^{p}$ norms for eigenfunctions of differential elliptic operators on general closed manifolds has been studied extensively; see, e.g., [SZ02]. Some examples are given at the end of this section. An example can be also obtained from the following simple lemma.

Lemma 8.2. Let $f$ be such that $\|f\|_{L^{2}(M)}=1$. Then

(i) $\|f\|_{L^{p}(M)} \leq(\operatorname{vol}(M))^{(2-p) / 2 p}$ if $1 \leq p \leq 2$;

(ii) $\|f\|_{L^{p}(M)} \leq\|f\|_{L^{\infty}(M)}^{(p-2) / p}$ if $2 \leq p<\infty$.

Proof. (i) By Hölder inequality, we have

$$
\int_{M}|f(x)|^{p} d x \leq\left(\int_{M}|f(x)|^{p^{\frac{2}{p}}} d x\right)^{\frac{p}{2}}\left(\int_{M}|1|^{\frac{2}{2-p}} d x\right)^{\frac{2-p}{2}}=(\operatorname{vol}(M))^{\frac{2-p}{2}} .
$$


(ii) We also have

$$
\int_{M}|f(x)|^{p} d x=\int_{M}|f(x)|^{p-2}|f(x)|^{2} d x \leq\|f\|_{L^{\infty}(M)}^{p-2} .
$$

We now formulate a sufficient condition for the $r$-nuclearity on $L^{p}(M)$ spaces as an application of the notion of the matrix-symbol on closed manifolds. Inspired by Lemma 8.2, we use the following function $\tilde{p}$ for $1 \leq p \leq \infty$ :

$$
\tilde{p}:= \begin{cases}0 & \text { if } 1 \leq p \leq 2, \\ (p-2) / p & \text { if } 2<p<\infty \\ 1 & \text { if } p=\infty\end{cases}
$$

We denote the dual indices of $p_{1}, p_{2}$, respectively, by $q_{1}:=p_{1}^{\prime}$ and $q_{2}:=p_{2}^{\prime}$.

Theorem 8.3. Let $1 \leq p_{1}, p_{2}<\infty$ and $0<r \leq 1$. Let $T: L^{p_{1}}(M) \rightarrow L^{p_{2}}(M)$ be a strongly invariant linear continuous operator whose matrix-valued symbol $\sigma(\ell)$ satisfies

$$
\sum_{\ell=0}^{\infty} \sum_{m, k=1}^{d_{\ell}}\left|\sigma(\ell)_{m k}\right|^{r} \Lambda(\ell, m ; n, \infty)^{\tilde{p_{2}} r} \Lambda(\ell, k ; n, \infty)^{\tilde{q_{1}} r}<\infty .
$$

Then the operator $T: L^{p_{1}}(M) \rightarrow L^{p_{2}}(M)$ is $r$-nuclear.

Proof. By (7.1), the kernel of $T$ is given by

$$
K(x, y)=\sum_{\ell=0}^{\infty} \sum_{m=1}^{d_{\ell}} \sum_{k=1}^{d_{\ell}} \sigma(\ell)_{m k} e_{\ell}^{m}(x) \overline{e_{\ell}^{k}(y)} .
$$

We set

$$
g_{\ell, m, k}(x):=\sigma(\ell)_{m k} e_{\ell}^{m}(x), h_{\ell, k}(y):=\overline{e_{\ell}^{k}(y)} .
$$

Now, by Lemma 8.2 ,

$$
\left\|e_{\ell}^{m}\right\|_{L^{p}} \leq C_{p} \Lambda(\ell, m ; n, \infty)^{\tilde{p}},
$$

where $C_{p}=\max \left\{(\operatorname{vol}(M))^{\frac{2-p}{2 p}}, 1\right\}$. We next observe that

$$
\begin{aligned}
\sum_{\ell, m, k}\left\|g_{\ell, m, k}\right\|_{L^{p_{2}}}^{r}\left\|h_{\ell, k}\right\|_{L^{q_{1}}}^{r}=\sum_{\ell=0}^{\infty} \sum_{m, k=1}^{d_{\ell}}\left\|\sigma(\ell)_{m k} e_{\ell}^{m}\right\|_{L^{p_{2}}}^{r}\left\|\overline{e_{\ell}^{k}}\right\|_{L^{q_{1}}}^{r} \\
\quad=\sum_{\ell=0}^{\infty} \sum_{m, k=1}^{d_{\ell}}\left|\sigma(\ell)_{m k}\right|^{r}\left\|e_{\ell}^{m}\right\|_{L^{p_{2}}}^{r}\left\|e_{\ell}^{k}\right\|_{L^{q_{1}}}^{r} \\
\leq\left(C_{p_{2}} C_{q_{1}}\right)^{r} \sum_{\ell=0}^{\infty} \sum_{m, k=1}^{d_{\ell}}\left|\sigma(\ell)_{m k}\right|^{r} \Lambda(\ell, m ; n, \infty)^{\tilde{p_{2} r}} \Lambda(\ell, k ; n, \infty)^{\tilde{q_{1} r}},
\end{aligned}
$$

finishing the proof in view of Theorem 8.1 . 
In particular, for formally self-adjoint invariant operators, we can diagonalise each matrix $\sigma(\ell)$, yielding the following corollary.

Corollary 8.4. Let $1 \leq p_{1}, p_{2}<\infty$ and $0<r \leq 1$. Suppose that $T: L^{p_{1}}(M) \rightarrow L^{p_{2}}(M)$ is a strongly invariant formally self-adjoint continuous operator whose matrix-valued symbol $\sigma(\ell)$ satisfies

$$
\sum_{\ell=0}^{\infty} \sum_{m=1}^{d_{\ell}}\left|\sigma(\ell)_{m m}\right|^{r} \Lambda(\ell, m ; n, \infty)^{\left(\tilde{p_{2}}+\tilde{q_{1}}\right) r}<\infty .
$$

Then the operator $T: L^{p_{1}}(M) \rightarrow L^{p_{2}}(M)$ is $r$-nuclear.

In some cases, it is possible to simplify the sufficient condition above when the control function $\Lambda(\ell, m ; n, \infty)$ is independent of $m$. For instance, a classical result (local Weyl law) of Hörmander ([Hör68, Theorem 5.1], [Hör85b, Chapter XXIX]) implies the following estimate.

Lemma 8.5. Let $M$ be a closed manifold of dimension $n$. Let $E \in \Psi_{+e}^{v}(M)$. Then

$$
\left\|e_{\ell}^{m}\right\|_{L^{\infty}} \leq C \lambda_{\ell}^{\frac{n-1}{2 v}}
$$

Proof. We first consider the family of eigenvalues $\left\{\lambda_{\ell}\right\}$ of $E$ in the increasing order

$$
0=\lambda_{0} \leq \lambda_{1} \leq \cdots \lambda_{\ell} \leq \cdots
$$

and counted with multiplicity. For the projection $P_{\ell}(f)$ onto $H_{\ell}$, the kernel of the associated partial sum operators $E_{\lambda} f:=\sum_{\lambda_{\ell} \leq \lambda} P_{\ell}(f)$ is given by

$$
E_{\lambda}(x, y)=\sum_{\lambda_{\ell} \leq \lambda} \sum_{m=1}^{d_{\ell}} e_{\ell}^{m}(x) \overline{e_{\ell}^{m}(y)}
$$

By [Hör68, Theorem 5.1],

$$
E_{\lambda}(x, x)=\sum_{\lambda_{\ell} \leq \lambda} \sum_{m=1}^{d_{\ell}}\left|e_{\ell}^{m}(x)\right|^{2}=(2 \pi)^{-n} \int_{p(x, \xi) \leq \lambda} d \xi+R(x, \lambda)
$$

with $|R(x, \lambda)| \leq C \lambda^{(n-1) / v}, x \in M$, where $p(x, \xi)$ is the principal symbol of $E$. Since $E_{\mu}(x, x)$ is increasing right-continuous with respect to $\mu$, by the fact that the spectrum of $E$ is discrete by the continuity of $\int_{p(x, \xi) \leq \mu} d \xi$ with respect to $\mu$, and by taking left-hand limit in 8.5 , we obtain

$$
\lim _{\mu \rightarrow \lambda^{-}} E_{\mu}(x, x)=\sum_{\lambda_{\ell}<\lambda} \sum_{m=1}^{d_{\ell}}\left|e_{\ell}^{m}(x)\right|^{2}=(2 \pi)^{-n} \int_{p(x, \xi) \leq \lambda} d \xi+R\left(x, \lambda^{-}\right) .
$$


Hence

$$
E_{\lambda_{\ell}}(x, x)-E_{\lambda_{\ell}}(x, x)=\sum_{m=1}^{d_{\ell}}\left|e_{\ell}^{m}(x)\right|^{2}=R\left(x, \lambda_{\ell}\right)-R\left(x, \lambda_{\ell}^{-}\right) .
$$

In particular,

$$
\left|e_{\ell}^{m}(x)\right| \leq 2\left(\sqrt{R\left(x, \lambda_{\ell}\right)}+\sqrt{R\left(x, \lambda_{\ell}^{-}\right)}\right) \leq 2 C \lambda_{\ell}^{\frac{n-1}{2 v}}
$$

Thus $\Lambda(\ell ; n, \infty)=C \lambda_{\ell}^{(n-1) / 2 v}$ furnishes an example of $\Lambda$ independent of $m$. For controls of type $\Lambda(\ell ; n, \infty)$, we have a basis-independent condition.

Corollary 8.6. Let $1 \leq p_{1}, p_{2}<\infty$ and $0<r \leq 1$. Suppose that $T: L^{p_{1}}(M) \rightarrow L^{p_{2}}(M)$ is a strongly invariant formally self-adjoint continuous operator whose matrix-valued symbol $\sigma(\ell)$ satisfies

$$
\sum_{\ell=0}^{\infty}\|\sigma(\ell)\|_{S_{r}}^{r} \Lambda(\ell ; n, \infty)^{\left(\tilde{p_{2}}+\tilde{q_{1}}\right) r}<\infty .
$$

Then the operator $T: L^{p_{1}}(M) \rightarrow L^{p_{2}}(M)$ is r-nuclear. In particular, if its matrixvalued symbol $\sigma(\ell)$ satisfies

$$
\sum_{\ell=0}^{\infty}\|\sigma(\ell)\|_{S_{r}}^{r} \lambda_{\ell}^{\frac{(n-1)}{2 v}\left(\tilde{p_{2}}+\tilde{q_{1}}\right) r}<\infty
$$

then the operator $T: L^{p_{1}}(M) \rightarrow L^{p_{2}}(M)$ is r-nuclear.

Proof. Since $T$ is $E$-invariant and formally self-adjoint, each matrix $\sigma(\ell)$ can be assumed diagonal. The result then follows from Corollary 8.4 , since

$$
\sum_{m=1}^{d_{\ell}}\left|\sigma(\ell)_{m m}\right|^{r}=\operatorname{Tr}\left(|\sigma(\ell)|^{r}\right)=\|\sigma(\ell)\|_{S_{r}}^{r} .
$$

The $r$-nuclearity under condition $(8.6)$ follows from Lemma 8.5 with $\Lambda(\ell ; n, \infty)=$ $C \lambda_{\ell}^{(n-1) / 2 v}$.

Remark 8.7. If $M$ is a compact Lie group, with $E$ taken to be the Laplacian and the family of eigenfunctions $\left\{e_{\ell}^{k}\right\}$ as in (6.7), Corollary 8.6 encompasses $\left[\right.$ DR14b, Theorem 3.4]. Indeed, since $\left|d_{\xi_{\ell}}^{\frac{1}{2}}\left(\xi_{\ell}\right)_{i j}(x)\right| \leq d_{\xi_{\ell}}^{1 / 2}$, one can choose $\Lambda(\ell ; \infty)=d_{\xi_{\ell}}^{1 / 2}$. Then, taking into account that $\|\sigma(\ell)\|_{S_{r}}^{r}=d_{\xi_{\ell}}\left\|\tau\left(\xi_{\ell}\right)\right\|_{S_{r}}^{r}$ by Lemma 6.1, we obtain

$$
\sum_{\ell}\|\sigma(\ell)\|_{S_{r}}^{r} \Lambda(\ell ; \infty)^{\left(\tilde{p_{2}}+\tilde{q_{1}}\right) r}=\sum_{\ell} d_{\xi_{\ell}}^{1+\frac{1}{2}\left(\tilde{p_{2}}+\tilde{q_{1}}\right) r}\left\|\tau\left(\xi_{\ell}\right)\right\|_{S_{r}}^{r},
$$


with a right-hand side equivalent to the term giving the sufficient condition in [DR14b, Theorem 3.4]. Indeed,

$$
\begin{aligned}
\frac{1}{2}\left(\tilde{p_{2}}+\tilde{q_{1}}\right) & =\frac{1}{2}\left(1-\frac{2}{\max \left\{2, p_{2}\right\}}+1-\frac{2}{\max \left\{2, q_{1}\right\}}\right) \\
& =1-\frac{1}{\max \left\{2, q_{1}\right\}}-\frac{1}{\max \left\{2, p_{2}\right\}} \\
& =\frac{1}{\min \left\{2, p_{1}\right\}}-\frac{1}{\max \left\{2, p_{2}\right\}},
\end{aligned}
$$

which is the order obtained in [DR14b, Theorem 3.4] on compact Lie groups.

In order to give another example, we recall Proposition 5.3 with useful relations between the eigenvalues $\lambda_{j}$ and their multiplicities $d_{j}$. As a consequence of Corollary 8.6 and Proposition 5.3 , for the negative powers of the operator $E$ itself we obtain the following corollary.

Corollary 8.8. Let $1 \leq p_{1}, p_{2}<\infty$ and $0<r \leq 1$. Let $E \in \Psi_{+e}^{v}(M)$. If

$$
\alpha>\frac{n}{r}+\left(\tilde{p_{2}}+\tilde{q_{1}}\right) \frac{n-1}{2},
$$

then the operator $(I+E)^{-\frac{\alpha}{v}}: L^{p_{1}}(M) \rightarrow L^{p_{2}}(M)$ is $r$-nuclear.

Note that if $p_{1}=p_{2}=2$, then $\tilde{p_{2}}=\tilde{q_{1}}=0$; and, since Schatten class $S_{r}$ and $r$-nuclear class coincide on $L^{2}(M)$, Proposition 5.4 shows that the statement of Corollary 8.8 is sharp in this case of indices. However, it does depend on the bounds for eigenvalues, which can be improved in the presence of additional structures, as discussed in Remark 8.9 .

Proof of Corollary 8.8. Denote by $\lambda_{\ell}$ the eigenvalues of $E$, Then, for $\alpha>0, \sigma_{(I+E)^{-\frac{\alpha}{v}}}(\ell)=\left(1+\lambda_{\ell}\right)^{-\frac{\alpha}{\nu}} I_{d_{\ell}}$. Thus

$$
\left\|\sigma_{(I+E)^{-\frac{\alpha}{v}}}(\ell)\right\|_{S_{r}}^{r}=\left(1+\lambda_{\ell}\right)^{-\frac{\alpha r}{v}} d_{\ell} .
$$

Now, applying Corollary 8.6 , by Proposition 5.3 , we obtain

$$
\begin{aligned}
\sum_{\ell}\|\sigma(\ell)\|_{S_{r}}^{r} \lambda_{\ell}^{\frac{(n-1)}{2 v}\left(\tilde{p_{2}}+\tilde{q_{1}}\right) r} & \leq C \sum_{\ell} d_{\ell}\left(1+\lambda_{\ell}\right)^{-\frac{\alpha r}{v}}\left(1+\lambda_{\ell}\right)^{\left(\tilde{p_{2}}+\tilde{q_{1}}\right) \frac{(n-1) r}{2 v}} \\
& =C \sum_{\ell} d_{\ell}\left(1+\lambda_{\ell}\right)^{\left(-\alpha+\left(\tilde{p_{2}}+\tilde{q_{1}}\right) \frac{(n-1)}{2}\right) \frac{r}{v}}<\infty,
\end{aligned}
$$

if

$$
q=\left(\alpha-\left(\tilde{p_{2}}+\tilde{q_{1}}\right) \frac{(n-1)}{2}\right) \frac{r}{v}>\frac{n}{v} .
$$


But this is equivalent to the condition

$$
\alpha>\frac{n}{r}+\left(\tilde{p_{2}}+\tilde{q_{1}}\right) \frac{n-1}{2} .
$$

Remark 8.9. As pointed out in Remark 8.7, on compact Lie groups, we can always choose $E$ to be a Laplacian with an orthonormal basis given by rescaled matrix elements of representations, for which we can take $\Lambda(\ell ; \infty)=d_{\xi_{\ell}}^{1 / 2}=d_{\ell}^{1 / 4}$. At the same time, if $E$ is an operator of second order (so that $v=2$ ), the best we can hope for on closed manifolds in general is $\Lambda(\ell ; n, \infty)=C \lambda_{\ell}^{(n-1) / 4}$, given by Lemma 8.5. In view of (5.1), we always have $d_{\ell}^{1 / 4} \lesssim \lambda_{\ell}^{n / 8}$, so that this choice on compact Lie groups is better than the general bound $\Lambda(\ell ; n, \infty)=C \lambda_{\ell}^{(n-1) / 4}$ above. This is explained partly by the presence of the additional (group) structure in this case. The other point is that there is a difference in finding $L^{\infty}$-estimates for elements of any orthonormal basis as opposed to estimates for a favourable one that may exist due to additional assumptions or structures. However, the latter one seems to be the question much less studied in the literature; see [SZ02] or [TZ02] for some partial discussions.

We now give an example of the above remark in the case of the the sphere $\mathbb{S}^{3} \simeq \mathrm{SU}(2)$. We consider the Laplacian (the Casimir element) $E=-\mathcal{L}_{\mathbb{S}^{3}}$. We apply the condition given by Theorem 8.6 , along with the control $\Lambda(\ell, \infty)=d_{\ell}^{1 / 4}$. For the symbol of $(I+E)^{-\frac{\alpha}{2}}$, since the eigenvalues of $I+E$ are of the form $(1+\ell) \ell$, we obtain

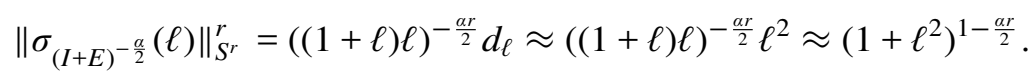

Therefore, using $d_{\ell} \approx \ell^{2}$, we have

$$
\begin{aligned}
\sum_{\ell}\left\|\sigma_{(I+E)^{-\frac{\alpha}{2}}}(\ell)\right\|_{S^{r}}^{r} \Lambda(\ell, \infty)^{\left(\tilde{p_{2}}+\tilde{q_{1}}\right) r} & \leq \sum_{\ell}\left(1+\ell^{2}\right)^{1-\frac{\alpha r}{2}} \ell^{\frac{1}{2}\left(\tilde{p_{2}}+\tilde{q_{1}}\right) r} \\
& \approx \sum_{\ell}(1+\ell)^{2-\alpha r+\frac{1}{2}\left(\tilde{p_{2}}+\tilde{q_{1}}\right) r} .
\end{aligned}
$$

The series on the right-hand side converges if and only if $2-\alpha r+\frac{1}{2}\left(\tilde{p_{2}}+\tilde{q_{1}}\right) r<-1$. Thus, the condition

$$
\alpha>\frac{3}{r}+\frac{1}{2}\left(\tilde{p_{2}}+\tilde{q_{1}}\right)
$$

ensures the membership of $(I+E)^{-\alpha / 2}$ in the Schatten class of order $r$. In summary, we have proved the following corollary. 
Corollary 8.10. If $\alpha>\frac{3}{r}+\frac{1}{2}\left(\tilde{p_{2}}+\tilde{q_{1}}\right), 0<r \leq 1$, the operator $\left(I-\mathcal{L}_{\mathbb{S}^{3}}\right)^{-\frac{\alpha}{2}}$ is $r$-nuclear from $L^{p_{1}}\left(\mathbb{S}^{3}\right)$ into $L^{p_{2}}\left(\mathbb{S}^{3}\right)$.

Corollary 8.10 gives a direct proof of [DR14b, Corollary 3.19], which was proved there in the group setting.

Remark 8.11. It is clear that the sharpness of the sufficient conditions obtained in this section depends on the sharpness in the $\Lambda$-function we can choose. For instance, the best situation for $\Lambda(\ell, \infty)$ is when it can be chosen to be constant, i.e., when the eigenfunctions are uniformly bounded. This is the case for the torus $\mathbb{T}^{n}$ which, unfortunately may be essentially the only case; see [TZ02].

Acknowledgements. We thank Véronique Fischer, Alexandre Kirilov, and Wagner Augusto Almeida de Moraes for comments.

Open Access. This article is distributed under the terms of the Creative Commons Attribution License which permits any use, distribution, and reproduction in any medium, provided the original author(s) and the source are credited.

\section{REFERENCES}

[Ati68] M. F. Atiyah, Global aspects of the theory of elliptic differential operators, in Proc. Internat. Congr. Math. (Moscow, 1966), Izdat. "Mir”, Moscow, 1968, pp. 57-64.

[BBR96] P. Boggiatto, E. Buzano, and L. Rodin, Global Hypoellipticity and Spectral Theory, Akademie Verlag, Berlin, 1996.

[BN04] E. Buzano and F. Nicola, Pseudo-differential operators and Schatten-von Neumann classes, in Advances in Pseudo-differential Operators, Birkhäuser, Basel, 2004, pp. 117-130.

[BN07] E. Buzano and F. Nicola, Complex powers of hypoelliptic pseudodifferential operators, J. Funct. Anal. 245 (2007), 353-378.

[BP61] A. Benedek and R. D. Panzone, The spaces $L^{p}$, with mixed norms, Duke Math. J. 28 (1961), 301-324.

[Bru68] F. Bruhat, Lectures on Lie Groups and Representations of Locally Compact Groups, Tata Institute of Fundamental Research, Bombay, 1968.

[BT10] E. Buzano and J. Toft, Schatten-von Neumann properties in the Weyl calculus, J. Funct. Anal. 259 (2010), 3080-3114.

[Car16] T. Carleman, Über die Fourierkoeffizienten einer stetigen Funktion, Acta Math. 41 (1916), 377-384.

[Cho11] O. Chodosh, Infinite matrix representations of isotropic pseudodifferential operators, Methods Appl. Anal. 18 (2011), 351-371.

[Del10] J. Delgado, The trace of nuclear operators on $L^{p}(\mu)$ for $\sigma$-finite Borel measures on second countable spaces, Integral Equations Operator Theory 68 (2010), 61-74.

[Dix77] J. Dixmier, $C^{*}$-algebras. North-Holland Publishing Co., Amsterdam-New York-Oxford, 1977. 
[Dix96] J. Dixmier, Les algèbres d'opérateurs dans l'espace hilbertien (algèbres de von Neumann), Éditions Jacques Gabay, Paris, 1996. Reprint of the second (1969) edition.

[DR14a] J. Delgado and M. Ruzhansky, Kernel and symbol criteria for Schatten classes and $r$ nuclearity on compact manifolds, C. R. Math. Acad. Sci. Paris 352 (2014), 779-784.

[DR14b] J. Delgado and M. Ruzhansky, $L^{p}$-nuclearity, traces, and Grothendieck-Lidskii formula on compact Lie groups, J. Math. Pures Appl. (9) 102 (2014), 153-172.

[DR14c] J. Delgado and M. Ruzhansky, Schatten classes on compact manifolds: kernel conditions, J. Funct. Anal. 267 (2014), 772-798.

[DR13] J. Delgado and M. Ruzhansky, Schatten classes and traces on compact groups, Math. Res. Lett. 24 (2017), 979-1003.

[Dui11] J. J. Duistermaat, Fourier Integral Operators. Birkhäuser/Springer, New York, 2011.

[GK69] I. C. Gohberg and M. G. Kreĭn, Introduction to the Theory of Linear Nonselfadjoint Operators. American Mathematical Society, Providence, RI, 1969.

[Gro55] A. Grothendieck, Produits tensoriels topologiques et espaces nucléaires American Mathematical Society, Providence, RI, 1955.

[GW73] S. J. Greenfield and N. R. Wallach, Remarks on global hypoellipticity Trans. Amer. Math. Soc. 183 (1973), 153-164.

[Hör68] L. Hörmander. The spectral function of an elliptic operator, Acta Math. 121 (1968), 193218.

[Hör85a] L. Hörmander. The Analysis of Linear Partial Differential Operators, vol. III, SpringerVerlag, 1985.

[Hör85b] L. Hörmander. The Analysis of Linear Partial Differential Operators, vol. IV, SpringerVerlag, 1985.

[HP10] A. Hinrichs and A. Pietsch, p-nuclear operators in the sense of Grothendieck, Math. Nachr. 283 (2010), 232-261.

[Kön78] H. König, Eigenvalues of p-nuclear operators, in Proceedings of the International Conference on Operator Algebras, Ideals, and their Applications in Theoretical Physics (Leipzig, 1977), Teubner, Leipzig, 1978, pp. 106-113.

[NR10] F. Nicola and L. Rodino, Global Pseudo-differential Calculus on Euclidean Spaces, Birkhäuser Verlag, Basel, 2010.

[Olo72] R. Oloff, p-normierte Operatorenideale, Beiträge Anal. 4 (1972), 105-108.

[Pie84] A. Pietsch, Grothendieck's concept of a p-nuclear operator, Integral Equations Operator Theory, 7 (1984), 282-284.

[RL13] O. I. Reinov and Q. Laif, Grothendieck-Lidskii theorem for subspaces of Lp-spaces, Math. Nachr. 2-3 (2013), 279-282.

[RS75] M. Reed and B. Simon, Methods of Modern Mathematical Physics II: Fourier Analysis, Self-adjointness, Academic Press, New York, 1975.

[RS80] M. Reed and B. Simon. Methods of Modern Mathematical Physics I: Functional Analysis, second edition, Academic Press, Inc., New York, 1980.

[RT10] M. Ruzhansky and V. Turunen, Pseudo-differential Operators and Symmetries. Background Analysis and Advanced Topics, Birkhäuser Verlag, Basel, 2010.

[RT13] M. Ruzhansky and V. Turunen, Global quantization of pseudo-differential operators on compact Lie groups, SU(2), 3-sphere, and homogeneous spaces, Int. Math. Res. Not. IMRN 2013 (2013), 2439-2496.

[RT15] M. Ruzhansky and N. Tokmagambetov, Nonharmonic analysis of boundary value problems, Int. Math. Res. Not. IMRN 2016, (2016), 3548-3615.

[Rus74] B. Russo, The norm of the Lp-Fourier transform on unimodular groups, Trans. Amer. Math. Soc. 192 (1974), 293-305. 
[Sch70] R. Schatten, Norm Ideals of Completely Continuous Operators, Springer-Verlag, Berlin, 1970.

[See65] R. T. Seeley, Integro-differential operators on vector bundles, Trans. Amer. Math. Soc. 117 (1965), 167-204.

[See67] R. T. Seeley. Complex powers of an elliptic operator, in Singular Integrals, Amer. Math. Soc., Providence, RI, 1967, pp. 288-307.

[See69] R. T. Seeley. Eigenfunction expansions of analytic functions, Proc. Amer. Math. Soc. 21 (1968), 734-738, 1969.

[Shu01] M. A. Shubin. Pseudodifferential Operators and Spectral Theory, second edition, SpringerVerlag, Berlin, 2001.

[Sim79] B. Simon, Trace Ideals and their Applications, Cambridge University Press, Cambridge, 1979.

[Sob14] A. V. Sobolev, On the Schatten-von Neumann properties of some pseudo-differential operators, J. Funct. Anal. 266 (2014), 5886-5911.

[Ste70] E. M. Stein, Topics in Harmonic Analysis Related to the Littlewood-Paley Theory, Princeton University Press, Princeton, NJ, 1970.

[Str72] R. S. Strichartz, A functional calculus for elliptic pseudo-differential operators, Amer. J. Math. 94 (1972), 711-722.

[SZ02] C. Sogge and S. Zelditch, Riemannian manifolds with maximal eigenfunction growth, Duke Math. J. 114 (2002), 387-437.

[Tof06] J. Toft, Schatten-von Neumann properties in the Weyl calculus, and calculus of metrics on symplectic vector spaces, Ann. Global Anal. Geom. 30 (2006), 169-209.

[Tof08] J. Toft, Schatten properties for pseudo-differential operators on modulation spaces, in Pseudo-differential Operators, Springer, Berlin, 2008, pp. 175-202.

[TZ02] J. Toth and S. Zelditch, Riemannian manifolds with uniformly bounded eigenfunctions, Duke Math. J. 111 (2002), 97-132.

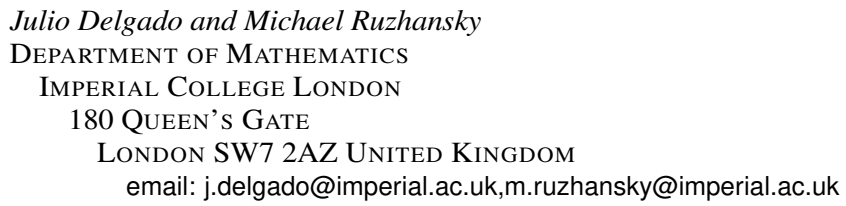

(Received November 6, 2015) 